\title{
A dynamic-solver-consistent minimum action method: With an application to 2D Navier-Stokes equations
}

\author{
Xiaoliang Wan ${ }^{\mathrm{a}, *}$, Haijun $\mathrm{Yu}^{\mathrm{b}}$ \\ ${ }^{a}$ Department of Mathematics and Center for Computation and Technology, \\ Louisiana State University, Baton Rouge 70803 \\ ${ }^{b}$ NCMIS \& LSEC, Institute of Computational Mathematics and Scientific/Engineering \\ Computing, Academy of Mathematics and Systems Science, Beijing 100190, China
}

\begin{abstract}
This paper discusses the necessity and strategy to unify the development of a dynamic solver and a minimum action method (MAM) for a spatially extended system when employing the large deviation principle (LDP) to study the effects of small random perturbations. A dynamic solver is used to approximate the unperturbed system, and a minimum action method is used to approximate the LDP, which corresponds to solving an Euler-Lagrange equation related to but more complicated than the unperturbed system. We will clarify possible inconsistencies induced by independent numerical approximations of the unperturbed system and the LDP, based on which we propose to define both the dynamic solver and the MAM on the same approximation space for spatial discretization. The semi-discrete LDP can then be regarded as the exact LDP of the semi-discrete unperturbed system, which is a finite-dimensional ODE system. We achieve this methodology for the two-dimensional Navier-Stokes equations using a divergence-free approximation space. The method developed can be used to study the nonlinear instability of wall-bounded parallel shear flows, and be generalized straightforwardly to three-dimensional cases. Numerical experiments are presented.
\end{abstract}

Keywords: Minimum action method, Rare events, White noise, Colored noise, Finite element method, Numerical adaptivity.

\section{Introduction}

During the past two decades, there has been a widespread interest in uncertainty quantification (UQ) to develop stochastic models and approaches to quantitatively describe the propagation of uncertainty of different sources in

\footnotetext{
* Corresponding author

Email addresses: xlwan@math.lsu.edu (Xiaoliang Wan), hyu@lsec.cc.ac.cn (Haijun $\mathrm{Yu})$
}

Preprint submitted to Elsevier

October 26, 2016

(C) 2016. This manuscript version is made available under the Elsevier user license http://www.elsevier.com/open-access/userlicense/1.0/ 
complex systems, and the interplay of mathematical modeling and experimental data. Thanks to the development of stochastic numerics and high performance computing, many successes have been achieved in UQ by recasting some classical deterministic models in a stochastic setting. For example, classical deterministic partial differential equations (PDEs) have been relaxed to a random one by taking into account the uncertainty in physical parameters, initial/boundary conditions, etc. Bayesian inference has been applied to deal with inverse problems in spatially extended systems. Although a deterministic inverse problem is, in general, ill-posed, the noisy physical observations provide a natural feedback to the mathematical model in the probabilistic sense, which makes the stochastic inverse problem well-posed.

In this paper we are interested in generalizing a deterministic spatially extended system by introducing a small random forcing term, which results in a stochastic PDE (see equation (1)). In particular, we pay attention to transitions induced by the small noise. The importance of this problem is at least twofold: First, since the noise amplitude is small, the transition mechanism is closely related to the structure of the phase space of the deterministic system. For example, in gradient systems, the transition rate is determined by the saddle points of index one, which makes the search for saddle points a critical issue $[26,13,56,41,18]$. Second, such a stochastic model can be more appropriate to describe the nonlinear instability induced by the subcritical bifurcation. For example, in wall-bounded parallel shear flows, two or more stable solutions, including the laminar base flow, can exist for the same Reynolds number, where deterministic approaches, e.g., the linear stability theory, may not be effective to depict the instability of the base flow. Small noise in the forcing term, which can be regarded as background noise in experiments, establishes a random walk between the metastable solutions, which provides a natural probabilistic description of the nonlinear instability of the base flow $[14,52,55]$.

One important technique to study the small-noise-induced transitions is to employ the Frendlin-Wentzell theory of large deviations, where the central task is to minimize the so-called action functional, see equations (4). The minimizer is the most probable transition path, which, by the large deviation principle (LDP), provides an estimate of the transition probability in the asymptotic sense. Depending on the structure of the phase space and the definition of transitions, the difficulties of minimizing the action functional (or approximating the LDP) may vary (see [20] for a review of the instanton method, which summarizes some scenarios in the numerical approximation of large deviation principle). We here focus on the problem defined by the transition between any two points in the phase space (see equations (3) and (5)), where the EulerLagrange equation of the action functional defines a boundary value problem on a space-time domain (see equation (12)). In [11], this problem was first studied numerically by the so-called minimum action method (MAM). Since then, many versions of MAM have been developed, including the adaptive MAM [58], the geometric MAM [27], the high-order MAM [47, 48], the MAM with optimal linear time scaling [50], the $h p$-adaptive MAM [51], etc.

Except for a few works on string methods or MAMs with constraints (e.g. 
$[7,9,57,36])$, almost all the available MAMs focus on the numerical difficulties from the time direction. In this work, we will pay more attention to the spatial discretization in the application of the LDP to spatially extended systems, where we use a dynamic solver to approximate the unperturbed (or deterministic) system and the minimum action method (MAM) to approximate the LDP. One fundamental issue emerges when we treat a numerical trajectory given by a dynamic solver as a transition path for the LDP. If the nonlinear differentiation operator (see $\mathcal{G}$ in equation (1)) is not discretized in a consistent way for the dynamic solver and the action functional, the numerical LDP cannot discriminate between the residual induced by finite dimensional approximation and the action induced by external random forcing. This implies that a numerical transition path of zero action does not corresponds to a numerical trajectory given by the dynamic solver, although they are the same theoretically. We then propose a methodology to approximate the LDP and the unperturbed system using the same spatial discretization, where the semi-discrete LDP can be regarded as the exact LDP of the semi-discretized unperturbed system. We achieve this methodology for two-dimensional Navier-Stokes (N-S) equations using a divergence-free space. Compared to the MAM for N-S equations developed in [49], the current approach has several advantages:

- Inconsistency between the dynamic solver and the LDP in spatial discretization has been eliminated.

- The pressure has been removed from the formulation due to the projection onto a divergence-free space, i.e., the Helmholtz-Hodge decomposition. Thus, the number of degrees of freedom is reduced by half for the twodimensional N-S equations.

- The nonlinearity of the integrand of the action functional reduces from fourth order to second order, such that less quadrature points are needed to deal with the aliasing error.

This paper is organized as follows. In Section 2, we describe our target problem, where we clarify the possible discrepancies between numerical approximations of the LDP and the unperturbed system. In Section 3, we propose to use the same spatial discretization to define the minimum action method and the dynamic solver. We achieve our methodology for two-dimensional NavierStokes equations in Section 4. Numerical experiments are given in Section 5 followed by a summary section.

\section{Problem}

We start with the following general stochastic PDE:

$$
\partial_{t} u(t, \boldsymbol{x})+\mathcal{G}(u)(t, \boldsymbol{x})=\sqrt{\varepsilon} \dot{W}(t, \boldsymbol{x}),
$$

where $u(t, \boldsymbol{x}): \mathbb{R}^{+} \times D \mapsto \mathbb{R}, \boldsymbol{x} \in D \subset \mathbb{R}^{d}, d=1,2,3, \mathcal{G}$ a (nonlinear) spatial differentiation operator, $\varepsilon$ a small positive number and $\dot{W}(t, \boldsymbol{x})$ a Gaussian Hilbert 
space valued random process that is white in time and colored in space. Let $\mathcal{Q}$ be the spatial covariance operator, which is usually assumed to be symmetric positive and of trace class. When $\varepsilon=0$, equation (1) becomes a deterministic one:

$$
\partial_{t} u_{\mathrm{d}}(t, \boldsymbol{x})+\mathcal{G}\left(u_{\mathrm{d}}\right)(t, \boldsymbol{x})=0,
$$

where the subscript $\mathrm{d}$ indicates that $u_{\mathrm{d}}$ is a deterministic solution.

The effect of noise on dynamics is of particular interest especially when $\varepsilon$ is small, i.e., $0<\varepsilon \ll 1$. The fundamental difference between the stochastic dynamics given by equation (1) and the deterministic dynamics given by equation (2) is that there exist transitions between any two points in the phase space when noise exists. For small noise, the noise-induced transitions can be rigorously characterized by the large deviation principle (LDP) through the quasi-potential defined as:

$$
W\left(u_{1}, u_{2}\right)=S_{T^{*}}\left(u^{*}\right)=\inf _{T>0} \inf _{\substack{u(0, \boldsymbol{x})=u_{1}(\boldsymbol{x}), u(T, \boldsymbol{x})=u_{2}(\boldsymbol{x})}} S_{T}(u) .
$$

Here $T^{*}$ and $u^{*}$ indicate the minimizers, and $S_{T}(u)$ is called the action functional

$$
S_{T}(u)=\frac{1}{2} \int_{0}^{T}\left\langle\partial_{t} u+\mathcal{G}(u), \mathcal{Q}^{-1}\left(\partial_{t} u+\mathcal{G}(u)\right)\right\rangle_{D} d t,
$$

where $\langle\cdot, \cdot\rangle_{D}$ indicates the inner product in the Hilbert space on which the noise is defined. We call $u^{*}$ the minimal action path (MAP). The quasi-potential measures the difficulty of the passage from state $u_{1}$ to the vicinity of state $u_{2}$ in the following probabilistic sense [16]

$$
\lim _{\varepsilon \downarrow 0}-\varepsilon \log \operatorname{Pr}\left(\text { transitions from } u_{1} \text { to the vicinity of } u_{2}\right)=W\left(u_{1}, u_{2}\right) .
$$

The LDP also holds for a fixed time scale, i.e.,

$$
\begin{aligned}
& \lim _{\varepsilon \downarrow 0}-\varepsilon \log \operatorname{Pr}\left(\text { transitions from } u_{1} \text { to the vicinity of } u_{2} \text { at time } T\right) \\
& =S_{T}\left(u_{T}^{*}\right)=\inf _{\substack{u(0, \boldsymbol{x})=u_{1}(\boldsymbol{x}), u(T, \boldsymbol{x})=u_{2}(\boldsymbol{x})}} S_{T}(u) .
\end{aligned}
$$

The central task for the application of LDP is to solve the optimization problems in equations (3) and (5).

To approximate the deterministic equation (2) we need to specify an approximation space $V_{h}$ and look for $u_{\mathrm{d}} \approx u_{\mathrm{d}, h} \in V_{h}$ using a certain numerical procedure. To approximate the LDP we also need to choose an approximation space $\hat{V}_{h}$ and look for $u^{*} \approx u_{h}^{*} \in \hat{V}_{h}$. The approximation of LDP can be completely independent of the approximation of deterministic system (2). In this paper, we will refer to the solution of an unperturbed system as a trajectory, e.g., $u_{\mathrm{d}}$ and $u_{\mathrm{d}, h}$, and the functions studied by the LDP as a transition path, e.g., $u^{*}$ and $u_{h}^{*}$. 
One immediate question is: Do we need a dynamic solver of equation (2) to study the numerical MAP $u_{h}^{*}$ ? The answer is yes. For example, if $u_{1}$ and $u_{2}$ are two attractors, $u_{h}^{*}$ must pass through the separatrix between $u_{1}$ and $u_{2}$. To locate the intersection of $u_{h}^{*}$ and the separatrix, we need to use the states on $u_{h}^{*}$ as initial conditions of system (2) and look at the convergence behavior. There exists one transition state on $u_{h}^{*}$, across which the solution of the dynamic solver will switch between $u_{1}$ and $u_{2}$. This is a widely used strategy when tracking the laminar-turbulent boundary [42].

We then have to face a more serious question: How can we guarantee that the approximation of LDP and the dynamic solver are consistent? For example, if $u_{1}$ and $u_{2}$ are located on a trajectory $u_{\mathrm{d}}$, we should have $u^{*}=u_{\mathrm{d}}$ theoretically. In other words, regarded as a transition path, a trajectory has a zero action, and is a minimizer of the action functional. However, this relation is difficult to hold exactly for the numerical approximations $u_{h}^{*}$ and $u_{\mathrm{d}, h}$. One possible issue is the residual $\epsilon_{r}$ :

$$
\partial_{t} u_{\mathrm{d}, h}+\mathcal{G}\left(u_{\mathrm{d}, h}\right)=\epsilon_{r} \neq 0,
$$

which is induced by the truncation of an infinite-dimensional space to a finitedimensional one, e.g., $V_{h}$. The residual $\epsilon_{r}$ defines the inconsistency between the finite-dimensional approximations of the unperturbed system and LDP. In a dynamic solver, $\mathcal{G}$ is often replaced by its projection onto the finite-dimensional approximation space, e.g., the Galerkin approach, to obtain the discrete equation; however, to discretize the action functional (4), $\mathcal{G}$ is not necessarily projected especially when an optimization solver is employed. When plugging $u_{\mathrm{d}, h}$ into the action functional, the residual $\epsilon_{r}$ is indistinguishable from action. If we choose two points on a numerical trajectory, it is possible that the approximated LDP yields a different path subject to a smaller "action". This way, the approximated LDP and the dynamic solver do not have an agreement on the definition of a numerical trajectory. More observations about the negative effect of such an issue can be found in [52], where the nonlinear instability of two-dimensional Poiseuille flow in a short channel was studied by the minimum action method.

Hence it is necessary to unify the approximations of the LDP and the deterministic system such that the inconsistencies induced by numerical discretization can be reduced to the minimum.

\subsection{Approximation of system (2)}

There exist numerous methods to approximate equation (2), including finite difference method, finite volume method, finite element method, spectral method, as well as many other numerical methods tied to the specific character of the equation. Different methods may employ different strategies for discretization, spatial discretization in particular. Here we consider methods based on the Galerkin projection.

Let $V_{h}$ be an approximation space defined on the physical domain $D$. Let $\mathcal{P}_{h}$ be a projection operator onto $V_{h}$ such that for a function $f(\boldsymbol{x}) \in L^{2}(D)$ we 
have $\mathcal{P}_{h} f \in V_{h}$, and

$$
\left\langle\mathcal{P}_{h} f, v(\boldsymbol{x})\right\rangle_{D}=\langle f(\boldsymbol{x}), v(\boldsymbol{x})\rangle_{D}, \quad \forall v \in V_{h}
$$

Then system (2) is often approximated in the weak sense through a certain type of projection, e.g., the Galerkin projection. In other words, we seek $u_{\mathrm{d}, h} \in V_{h}$, such that

$$
\left\langle\partial_{t} u_{\mathrm{d}, h}, v\right\rangle_{D}+\left\langle\mathcal{G}\left(u_{\mathrm{d}, h}\right), v\right\rangle_{D}=0, \quad \forall v \in V_{h} .
$$

This is the weak form of equation (2), where integration by parts can often be used such that the regularity requirement on $V_{h}$ is minimized. For example, if $\mathcal{G}=-\Delta$ is a Laplace operator, we only need that $V_{h}$ belongs to $H^{1}$ instead of $H^{2}$. We rewrite equation (8) as

$$
\partial_{t} u_{\mathrm{d}, h}+\mathcal{P}_{h} \mathcal{G}\left(u_{\mathrm{d}, h}\right)=0 .
$$

The residual induced by $u_{\mathrm{d}, h}$ can be written as

$$
\epsilon_{r}\left(u_{\mathrm{d}, h}\right)=\partial_{t} u_{\mathrm{d}, h}+\mathcal{G}\left(u_{\mathrm{d}, h}\right)=\left(\mathcal{G}-\mathcal{P}_{h} \mathcal{G}\right)\left(u_{\mathrm{d}, h}\right) .
$$

We here do not consider the error from the temporal discretization since different strategies will be used for system (2) and the LDP due to the fact that LDP defines a boundary value problem on a space-time domain (see equation (12)). We have also assumed in the definition of residual $\epsilon_{r}$ that $V_{h}$ has the regularity required by $\mathcal{G}$. Otherwise, we are not able to define $\mathcal{G}\left(u_{\mathrm{d}, h}\right)$. This is a strong assumption for the numerical solution of system (2). However, it allows us to use the same approximation space for both system (2) and the LDP. This issue will be further clarified in the next section.

\subsection{Approximation of $L D P$}

Let $\hat{V}_{h}$ the approximation space for the LDP. We seek $u_{h}^{*} \in \hat{V}_{h}$ such that

$$
S_{T_{h}^{*}}\left(u_{h}^{*}\right)=\min _{\substack{T \in \mathbb{R}^{+}, u_{h}(0)=u_{1}, u_{h}(T)=u_{2}}} \frac{1}{2} \int_{0}^{T}\left\langle\partial_{t} u_{h}+\mathcal{G}\left(u_{h}\right), \mathcal{Q}^{-1}\left(\partial_{t} u_{h}+\mathcal{G}\left(u_{h}\right)\right)\right\rangle_{D} d t .
$$

The minimizer can be obtained by gradient-type optimization algorithms or more efficient algorithms if the computation of Hessian is affordable, such as Newton's method. For a fixed integration time $T$, finding $u_{h}^{*}$ corresponds to approximate the following Euler-Lagrange equation

$$
\left(\partial_{t}+\hat{\mathcal{G}}\right)^{*} \mathcal{Q}^{-1}\left(\partial_{t}+\mathcal{G}\right)(u)=0, \quad(t, \boldsymbol{x}) \in[0, T] \times D,
$$

where the operator $\hat{\mathcal{G}}$ is given by the linearization of $\mathcal{G}$, and $\left(\partial_{t}+\hat{\mathcal{G}}\right)^{*}$ the adjoint operator of $\partial_{t}+\hat{\mathcal{G}}$.

Mathematically, $\hat{V}_{h}$ is often chosen to be different from $V_{h}$. This fact can be illustrated by assuming that $\mathcal{G}=-\Delta$, i.e., a Laplace operator. Then one weak from for $u_{\mathrm{d}, h}$ is

$$
\left\langle\partial_{t} u_{\mathrm{d}, h}, v\right\rangle_{D}+\langle\nabla u, \nabla v\rangle_{D}-\left\langle\partial_{\mathbf{n}} v, u\right\rangle_{\partial D}=0, \quad \forall v \in V_{h},
$$


where $\mathbf{n}$ indicates the outward pointing normal direction on the boundary $\partial D$ and we used integration by parts once. It is seen that this weak form requires that $V_{h} \subset H^{1}(D)$. The definition (4) of action functional implies that $\hat{V}_{h} \subset$ $H^{2}(D)$. In other words, the action functional requires a smoother approximation space. To remove such an inconsistency between $\hat{V}_{h}$ and $V_{h}$, the simplest way is to let $\hat{V}_{h}=V_{h}$, which, however, will directly affect the numerical procedure for both the LDP and system (2). This is not a trivial issue from the numerical point of view. We have two options to choose: (1) $V_{h}=\hat{V}_{h} \subset H^{2}(D)$; and (2) $V_{h}=\hat{V}_{h} \subset H^{1}(D)$. For the first option, we may need to reconsider the approximation of system (2); for the second choice, we have to reconsider the discretization of the LDP.

Both options are feasible. In this work, we will consider the first option motivated by a fundamental problem in fluid mechanics: nonlinear instability of wall-bounded parallel shear flows. For this problem, spectral methods are widely used for system (2) due to the simple geometry of the physical domain. Since orthogonal polynomials used in spectral methods are smooth functions, we can naturally let $V_{h}=\hat{V}_{h}$. We then consider this special case and leave the general case for future study.

We now quantify some errors to measure the inconsistencies induced by the discretization of system (2) and the LDP.

Proposition 1. Let the numerical MAP $u_{h}^{*}$ correspond to a trajectory of system (2). We have

$$
\left|\partial_{t} u_{h}^{*}+\mathcal{P}_{h} \mathcal{G}\left(u_{h}^{*}\right)\right| \leq \epsilon_{r}\left(u_{h}^{*}\right)+\left\|\mathcal{P}_{h} \mathcal{G}-\mathcal{G}\right\|\left\|u_{h}^{*}\right\| .
$$

Proof. We have

$$
\begin{aligned}
\left|\partial_{t} u_{h}^{*}+\mathcal{P}_{h} \mathcal{G}\left(u_{h}^{*}\right)\right| & =\left|\partial_{t} u_{h}^{*}+\mathcal{G}\left(u_{h}^{*}\right)+\left(\mathcal{P}_{h} \mathcal{G}-\mathcal{G}\right)\left(u_{h}^{*}\right)\right| \\
& \leq\left|\partial_{t} u_{h}^{*}+\mathcal{G}\left(u_{h}^{*}\right)\right|+\left\|\mathcal{P}_{h} \mathcal{G}-\mathcal{G}\right\|\left\|u_{h}^{*}\right\| .
\end{aligned}
$$

It is seen that the semi-discrete problem (11) may not provide a transition path that satisfy equation (9) exactly even if $\hat{V}_{h}=V_{h}$ and zero action is reached. This is because the semi-discrete problem (11) uses $\mathcal{G}$ instead of $\mathcal{P}_{h} \mathcal{G}$.

Proposition 2. Let $u_{\mathrm{d}, h}$ be a numerical trajectory given by equation (9). We have

$$
S_{T}\left(u_{\mathrm{d}, h}\right)=\frac{1}{2} \int_{0}^{T}\left\|\epsilon_{r}\left(u_{\mathrm{d}, h}\right)\right\|_{\mathcal{Q}^{-1}}^{2} d t,
$$

where $\|v\|_{\mathcal{Q}^{-1}}^{2}=\left\langle v, \mathcal{Q}^{-1} v\right\rangle_{D}$ defines a weighted norm of $v$, and $\epsilon_{r}$ is the residual defined in equation (10).

PROOF.

$$
\begin{aligned}
S_{T}\left(u_{\mathrm{d}, h}\right) & =\frac{1}{2} \int_{0}^{T}\left\|\left(\partial_{t}+\mathcal{P}_{h} \mathcal{G}+\mathcal{G}-\mathcal{P}_{h} \mathcal{G}\right)\left(u_{\mathrm{d}, h}\right)\right\|_{\mathcal{Q}^{-1}}^{2} d t \\
& =\frac{1}{2} \int_{0}^{T}\left\|\left(\mathcal{G}-\mathcal{P}_{h} \mathcal{G}\right)\left(u_{\mathrm{d}, h}\right)\right\|_{\mathcal{Q}^{-1}}^{2} d t=\frac{1}{2} \int_{0}^{T}\left\|\epsilon_{r}\left(u_{\mathrm{d}, h}\right)\right\|_{\mathcal{Q}^{-1}}^{2} d t
\end{aligned}
$$

which is positive as long as $\mathcal{G}\left(u_{d, h}\right) \neq \mathcal{P}_{h} \mathcal{G}\left(u_{d, h}\right)$. 
Thus a numerical trajectory given by equation (9) has a positive action, which is given by the accumulation of residuals.

Remark 1. If we decompose the space $\mathcal{G} V_{h}=V_{h} \oplus V_{h}^{\perp}$ with respect to $\|\cdot\|_{\mathcal{Q}^{-1}}$, and let $\mathcal{G}\left(u_{h}\right)=\mathcal{G}^{V}\left(u_{h}\right)+\mathcal{G}^{\perp}\left(u_{h}\right)$, where $\mathcal{G}^{V}\left(u_{h}\right) \in V_{h}$ and $\mathcal{G}^{\perp}\left(u_{h}\right) \in V_{h}^{\perp}$, we have

$$
S_{T}\left(u_{h}\right)=\frac{1}{2} \int_{0}^{T}\left\|\left(\partial_{t}+\mathcal{G}^{V}\right)\left(u_{h}\right)\right\|_{\mathcal{Q}^{-1}}^{2}+\left\|\mathcal{G}^{\perp}\left(u_{h}\right)\right\|_{\mathcal{Q}^{-1}}^{2} d t
$$

If $\mathcal{G}$ is nonlinear, $\left\|\left(\partial_{t}+\mathcal{G}^{V}\right)\left(u_{h}\right)\right\|_{\mathcal{Q}^{-1}}$ and $\left\|\mathcal{G}^{\perp}\left(u_{h}\right)\right\|_{\mathcal{Q}^{-1}}$ usually cannot be zero at the same time. Although the MAP $u^{*}$ should recover a trajectory when there exists dynamics between $u_{1}$ and $u_{2}$, the zero action, in general, cannot be reached by the semi-discrete action functional (11) due to the finite dimensional approximation. If the transition time $T$ is large, the accumulation of residuals might affect the search of the MAP [52].

\section{Choose a numerical plan for system (2) and LDP}

We seek a numerical plan that meets the following conditions: (1) The same spatial discretization is used for both system (2) and the LDP; and (2) the residual $\epsilon_{r}$ is restricted to temporal discretization only. Such a numerical plan is based on the fact that system (2) is an initial value problem while the EulerLagrange equation (12) is a boundary value problem.

\subsection{A semi-discrete system}

To achieve our goal, we choose to start from SPDE (1) instead of the definition of action functional. We look at the semi-discrete system

$$
\partial_{t} \hat{u}+\mathcal{P}_{h} \mathcal{G}(\hat{u})=\sqrt{\varepsilon} \mathcal{P}_{h} \dot{W},
$$

where $\hat{u}$ is a random element taking its value in $V_{h}$. As $\varepsilon \rightarrow 0$, the deterministic semi-discrete system (9) is obtained.

Remark 2. The semi-discrete system (15) has received much attention as an approximation of stochastic PDE (1) when $\mathcal{P}_{h}$ corresponds to a finite element approximation space and $\mathcal{G}$ is a linear differentiation operator (see $[25,45,54$, $8,28,32,33,34]$, to name a few).

Let $\left\{\left(\lambda_{h, i}, e_{h, i}(\boldsymbol{x})\right)\right\}_{i=1}^{N_{h}}$ be the numerical solution of the eigenvalue problem $\mathcal{Q} v=\lambda v$ given by the approximation space $V_{h}$, i.e.,

$$
\mathcal{Q}_{h} e_{h, i}=\lambda_{h, i} e_{h, i},
$$

where $\mathcal{Q}_{h}=\mathcal{P}_{h} \mathcal{Q} \mathcal{P}_{h}: V_{h} \rightarrow V_{h}$ and $N_{h}$ the cardinality of $V_{h}$. We then use

$$
W_{h}(t, \boldsymbol{x})=\sum_{i=1}^{N_{h}} \lambda_{h, i}^{1 / 2} \beta_{i}(t) e_{h, i}(\boldsymbol{x})
$$


to approximate $\mathcal{P}_{h} W$, where $\beta_{i}$ are mutually independent standard real-valued Brownian motions. It is easy to show that $W_{h}$ has a covariance operator $\mathcal{Q}_{h}$. We can then replace $\mathcal{P}_{h} W$ with $W_{h}$ and consider the stochastic system

$$
\partial_{t} \hat{u}+\mathcal{P}_{h} \mathcal{G}(\hat{u})=\sqrt{\varepsilon} \dot{W}_{h} .
$$

Since $\mathcal{Q}_{h}$ is symmetric and positive definite, we let $V_{h}=\operatorname{span}\left\{e_{h, i}\right\}_{i=1}^{N_{h}}$, where $\left\langle e_{h, i}, e_{h, j}\right\rangle_{D}=\delta_{i j}$. Equation (18) yields the following stochastic differential equation:

$$
d X_{h}+b\left(X_{h}\right) d t=\sqrt{\varepsilon} \Lambda d B,
$$

where $X_{h}=\left[\begin{array}{lll}\hat{u}_{1} & \hat{u}_{2} \ldots \hat{u}_{N_{h}}\end{array}\right]^{\top} \in \mathbb{R}^{N_{h}}$ consists of the expansion coefficients of $\hat{u}=$ $\sum_{i=1}^{N_{h}} \hat{u}_{i} e_{h, i},\left(b\left(X_{h}\right)\right)_{i}=\left\langle\mathcal{G} \hat{u}, e_{h, i}\right\rangle_{D}, i=1, \ldots, N_{h}, \Lambda=\operatorname{diag}\left(\lambda_{h, 1}^{1 / 2}, \ldots, \lambda_{h, N_{h}}^{1 / 2}\right)$, and $B=\left[\beta_{1} \beta_{2} \ldots \beta_{N_{h}}\right]^{\top}$. Since $\Lambda \Lambda^{\top}$ is symmetric and positive definite (SPD), the action functional for a path $\psi(t) \in \mathbb{R}^{N_{h}}$ can be defined as

$$
S_{T}(\psi)=\frac{1}{2} \int_{0}^{T}(\dot{\psi}+b(\psi))^{\top} \Lambda^{-\top} \Lambda^{-1}(\dot{\psi}+b(\psi)) d t,
$$

where $\Lambda^{-\mathrm{T}} \Lambda^{-1}=\operatorname{diag}\left(\lambda_{h, 1}^{-1}, \ldots, \lambda_{h, N_{h}}^{-1}\right)$. On the other hand, we can regard equation (18) as an evolution equation of a Hilbert space valued random variable, which yields a action functional for $\hat{u}(t, \boldsymbol{x}) \in[0, T] \times D$ on $V_{h}$

$$
S_{T}(\hat{u})=\frac{1}{2} \int_{0}^{T}\left\langle\partial_{t} \hat{u}+\mathcal{P}_{h} \mathcal{G}(\hat{u}), \mathcal{Q}_{h}^{-1}\left(\partial_{t} \hat{u}+\mathcal{P}_{h} \mathcal{G}(\hat{u})\right)\right\rangle_{D} d t
$$

In contrast to the action functional defined in equation (4), we refer to equation (21) as the truncated action functional. On $V_{h}$, we define

$$
\mathcal{Q}_{h}^{-1}=\sum_{i=1}^{N_{h}} \lambda_{h, i}^{-1}\left\langle\cdot, e_{h, i}\right\rangle_{D} e_{h, i} .
$$

We then obtain

$$
\begin{aligned}
\left\|\partial_{t} \hat{u}+\mathcal{P}_{h} \mathcal{G}(\hat{u})\right\|_{\mathcal{Q}_{h}^{-1}}^{2} & =\sum_{i=1}^{N_{h}} \lambda_{h, i}^{-1}\left\langle\partial_{t} \hat{u}+\mathcal{P}_{h} \mathcal{G}(\hat{u}), e_{h, i}\right\rangle_{D}^{2} \\
& =\sum_{i=1}^{N_{h}} \lambda_{h, i}^{-1}\left(\dot{\hat{u}}_{i}+\left\langle\mathcal{P}_{h} \mathcal{G}(\hat{u}), e_{h, i}\right\rangle_{D}\right)^{2} \\
& =\sum_{i=1}^{N_{h}} \lambda_{h, i}^{-1}\left(\dot{\hat{u}}_{i}+\left\langle\mathcal{G}(\hat{u}), e_{h, i}\right\rangle_{D}\right)^{2},
\end{aligned}
$$

which is consistent with the integrand in equation (20).

Remark 3. If $\mathcal{Q}=\mathcal{I}$, where $\mathcal{I}$ is an identity operator, the noise is space-time white. $\mathcal{I}$ does not have a finite trace on $L_{2}(D)$, and the stochastic PDE (1) is 
usually ill-posed especially when $d \geq 2$. However, the approximated noise $W_{h}$ has a covariance operator $\mathcal{Q}_{h}=\mathcal{P}_{h}^{2}$, which does have a finite trace. For this case, the norm $\|\cdot\|_{\mathcal{Q}_{h}^{-1}}$ becomes the regular $L_{2}$ norm. Recently, many efforts have been made to regularize the behavior induced by space-time white noise. One observation is that under certain conditions, such as $0<\varepsilon \ll l_{c} \ll 1$, where $l_{c}$ indicates the correlation length, the norm $\|\cdot\|_{\mathcal{Q}^{-1}}$ can be replaced with the regular $L_{2}$ norm $[5,2,24]$ in the sense that the minimizer of the action functional converges correspondingly as $\mathcal{Q}$ converges to $\mathcal{I}$, i.e., the correlation length $l_{c}$ goes to zero.

Remark 4. For a compact operator $\mathcal{Q}$, the eigenvalues will be clustered at zero. The problem $\mathcal{Q}^{-1} g=f$ is ill-posed since $\mathcal{Q}^{-1}$ is not continuous. Although all eigenvalues of $\mathcal{Q}_{h}$ are positive, they can decay fast to be less than the machine accuracy if the correlation length is relatively large. We then need regularization to deal with $Q_{h}^{-1}$. More discussions about this issue can be found in [53].

\subsection{Minimum action method (MAM)}

From now on, we consider the minimum action method for system (18) using the action functional defined in equation (21). In other words, we look at the following optimization problem

$$
S_{T_{h}^{*}}\left(u_{h}^{*}\right)=\min _{\substack{T \in \mathbb{R}^{+}, u_{h}(0)=u_{h, 1}, u_{h}(T)=u_{h, 2}}} \frac{1}{2} \int_{0}^{T}\left\|\partial_{t} u_{h}+\mathcal{P}_{h} \mathcal{G}\left(u_{h}\right)\right\|_{\mathcal{Q}_{h}^{-1}}^{2} d t,
$$

where we still use $T_{h}^{*}$ and $u_{h}^{*}$ to indicate the minimizers, and we assume that $u_{h, 1}$ and $u_{h, 2}$ are metastable attractors (or points on attractors of nonzero dimension) of the projected deterministic system (9).

To deal with the fact that $T^{*}$ may be equal to $\infty$, we will employ the MAM with optimal linear time scaling (or tMAM) [50]. More specifically, we consider a re-scaled time parameter $s=t / T \in \Gamma=[0,1]$ such that problem (23) can be rewritten as

$$
\min _{\substack{T \in \mathbb{R}^{+}, u_{h}(0)=u_{h, 1}, u_{h}(1)=u_{h, 2}}} \frac{T}{2} \int_{0}^{1}\left\|T^{-1} \partial_{s} u_{h}+\mathcal{P}_{h} \mathcal{G}\left(u_{h}\right)\right\|_{\mathcal{Q}_{h}^{-1}}^{2} d s .
$$

The optimality conditions include

$$
\frac{\partial S_{T}}{\partial T}=0, \quad \frac{\delta S_{T}}{\delta u_{h}}=0
$$

where we used functional derivative in the second equation. For any $u_{h}, \partial_{T} S_{T}=$ 0 has a unique solution at

$$
\hat{T}\left(u_{h}\right)=\left(\frac{\int_{0}^{1}\left\|\partial_{s} u_{h}\right\|_{\mathcal{Q}_{h}^{-1}}^{2} d s}{\int_{0}^{1}\left\|\mathcal{P}_{h} \mathcal{G}\left(u_{h}\right)\right\|_{\mathcal{Q}_{h}^{-1}}^{2} d s}\right)^{1 / 2} .
$$


We then enforce the optimality condition $\partial_{T} S_{T}=0$ in the formulation (24) to remove the optimization parameter $T$, and obtain a new formulation

$$
S_{\hat{T}\left(u_{h}^{*}\right)}\left(u_{h}^{*}\right)=\min _{\substack{u_{h}(0)=u_{h, 1}, u_{h}(1)=u_{h, 2}}} \frac{\hat{T}\left(u_{h}\right)}{2} \int_{0}^{1}\left\|\hat{T}^{-1}\left(u_{h}\right) \partial_{s} u_{h}+\mathcal{P}_{h} \mathcal{G}\left(u_{h}\right)\right\|_{\mathcal{Q}_{h}^{-1}}^{2} d s .
$$

The MAM with optimal linear scaling relies on the following observation:

Lemma 3 ([51]). Assume that $u_{h}$ is discretized by finite elements in s direction (see equation (46) for a definition of the approximation space). If $\hat{u}_{h}^{*}$ is the minimizer of the fully discretized version of problem (26), then

$$
\hat{T}\left(\hat{u}_{h}^{*}\right)<\infty .
$$

When $T_{h}^{*}$ is finite, we have $T_{h}^{*} \approx \hat{T}\left(\hat{u}_{h}^{*}\right)$. The main concern about the formulation (26) is that $T_{h}^{*}=\infty$. Lemma 3 shows that if we use finite elements for the temporal discretization of $u_{h}$, the fully discretized version of problem (26) is always well-posed in the sense that $\hat{T}\left(\hat{u}_{h}^{*}\right)<\infty$ although $T_{h}^{*}=\infty$. In other words, once problem (26) is discretized in time direction by finite elements, the numerical optimal integral time will become from $\infty$ to a finite number, and its value depends on the underlying finite element mesh. This way, we can consider a sequence of refined meshes to generate a minimizing sequence. As the mesh is refined, the numerical optimal integral time will increase towards infinity. In [51], we have developed numerical strategies for $h$ - or $h p$-adaptivity based on a posteriori error estimates, which can effectively recover the optimal convergence rate of finite element approximation.

\section{Two-dimensional wall-bounded parallel shear flows}

We consider the following two-dimensional stochastic Navier-Stokes (N-S) perturbation equations defined on a physical domain $(x, y) \in D=[0, L] \times$ $[-1,1]$ :

$$
\left\{\begin{aligned}
\frac{\partial \boldsymbol{u}}{\partial t}+\left(\boldsymbol{u}_{\mathrm{tot}} \cdot \nabla\right) \boldsymbol{u}_{\mathrm{tot}} & =-\nabla p+\frac{1}{R e} \Delta \boldsymbol{u}+\sqrt{\varepsilon} \dot{W}(t, \boldsymbol{x}), \\
\nabla \cdot \boldsymbol{u} & =0
\end{aligned}\right.
$$

where $L$ is the length of the channel, $R e$ is the Reynolds number, $\dot{W}$ indicates divergence-free noise that is white in time and colored in space, and $\varepsilon$ is a small positive number. Here and after $y$ is the wall-normal direction. Equation (27) is obtained as follows. We let $\boldsymbol{u}_{\text {tot }}=\boldsymbol{u}_{b}+\boldsymbol{u}$ and $p_{\text {tot }}=p_{b}+p$, where $\boldsymbol{u}_{b}$ and $p_{b}$ are the velocity and pressure of the 2D Poiseuille flow respectively. Then $\boldsymbol{u}$ and $p$ indicate the deviation from the base flow. Considering that $\boldsymbol{u}_{b}=\left(1-y^{2}, 0\right)$ and $p_{b}=-\frac{2}{R e} x$ and substituting $\boldsymbol{u}_{\text {tot }}$ and $p_{\text {tot }}$ into the original N-S equations, we obtain equation (27) as a simplification. We will consider no-slip boundary conditions $\boldsymbol{u}=0$ at $y= \pm 1$ and periodic conditions at $x=0, L$. We are interested in Poiseuille flows is because (more than) two stable states can exist 
for a Reynolds number located in a certain range, which implies that the noiseinduced transitions are important for the nonlinear instability of the base flow [52]. More specifically, when the Reynolds number $R e<R e_{G}$, the base flow is globally stable, where $R e_{G}$ indicates the onset of subcritical bifurcation; when $R e_{G}<R e<R e_{L}$, other sustainable states can exist besides the base flow, where $R e_{L}$ indicates the critical Reynolds number given by the linear stability theory; when $R e>R e_{L}$, the base flow is linearly unstable.

\subsection{Navier-Stokes equations defined on a divergence-free space}

Consider the divergence-free spaces

$$
\begin{aligned}
& H=\left\{\boldsymbol{u} \in L_{2}(D)|\nabla \cdot \boldsymbol{u}=0, \boldsymbol{u} \cdot \boldsymbol{n}=0|_{y= \pm 1},\left.\boldsymbol{u}\right|_{x=0}=\left.\boldsymbol{u}\right|_{x=L}\right\} \\
& V=\left\{\boldsymbol{u} \in H^{1}(D)|\nabla \cdot \boldsymbol{u}=0, \boldsymbol{u}=0|_{y= \pm 1},\left.\boldsymbol{u}\right|_{x=0}=\left.\boldsymbol{u}\right|_{x=L}\right\}
\end{aligned}
$$

Let the Wiener process $W(t)$ takes its value in $H$. Then the N-S equations can be written as the following stochastic evolution equation

$$
d \boldsymbol{u}+\Pi\left[\left(\boldsymbol{u}_{\mathrm{tot}} \cdot \nabla\right) \boldsymbol{u}_{\mathrm{tot}}-R e^{-1} \Delta \boldsymbol{u}\right] d t=\sqrt{\varepsilon} d W(t),
$$

where $\boldsymbol{u} \in V$ is divergence free, and $\Pi$ is a projection operator in $L_{2}(D)$ onto $H$ given by Helmholtz-Hodge decomposition, i.e., for any $\boldsymbol{u} \in L_{2}\left(D ; \mathbb{R}^{2}\right)$, there exists a unique $q \in H^{1}(D)$ with $\int_{D} q d \boldsymbol{x}=0$ such that $\Pi \boldsymbol{u}=\boldsymbol{u}+\nabla q$ satisfying

$$
0=\langle\Pi \boldsymbol{u}, \nabla \phi\rangle_{D}=\langle\boldsymbol{u}+\nabla q, \nabla \phi\rangle_{D}, \quad \phi \in H^{1}(D) .
$$

Since we only consider the divergence-free part, the pressure disappears in equation (28). The action functional for system (28) can be defined as [43]

$$
S_{T}(\boldsymbol{u})=\frac{1}{2} \int_{0}^{T}\left\|\partial_{t} \boldsymbol{u}-\Pi \mathcal{G}(\boldsymbol{u})\right\|_{\mathcal{Q}^{-1}}^{2} d t
$$

From now on, $\mathcal{G}(\boldsymbol{u})=\left(\boldsymbol{u}_{\text {tot }} \cdot \nabla\right) \boldsymbol{u}_{\text {tot }}-R e^{-1} \Delta \boldsymbol{u}$.

We now look at the truncated version of action functional (29). When $\varepsilon=0$, we have the deterministic system:

$$
\partial_{t} \boldsymbol{u}+\Pi \mathcal{G}(\boldsymbol{u})=0
$$

Let $V_{h} \subset V$ and $H_{h} \subset H$ be two approximation spaces of $V$ and $H$, respectively. We then seek $\boldsymbol{u}_{d, h} \in V_{h}$ such that

$$
\left\langle\partial_{t} \boldsymbol{u}_{d, h}, \boldsymbol{v}\right\rangle_{D}+\left\langle\Pi \mathcal{G}\left(\boldsymbol{u}_{d, h}\right), \boldsymbol{v}\right\rangle_{D}=0, \quad \boldsymbol{v} \in H_{h},
$$

where we assume $V_{h} \subseteq H_{h}$ since $V \subset H$. Note that due to the Helmholtz-Hodge decomposition, the spaces for trial functions and test functions can be different. We write equation (31) as

$$
\partial_{t} \boldsymbol{u}_{d, h}+\mathcal{P}_{h}^{\mathrm{H}} \mathcal{G}\left(\boldsymbol{u}_{d, h}\right)=0
$$


where $\mathcal{P}_{h}^{\mathrm{H}}=\mathcal{P}_{h} \Pi$ and $\mathcal{P}_{h}$ indicates the projection onto $H_{h}$. We then consider small random perturbations of system (32)

$$
\partial_{t} \boldsymbol{u}_{d, h}+\mathcal{P}_{h}^{\mathrm{H}} \mathcal{G}\left(\boldsymbol{u}_{d, h}\right)=\sqrt{\varepsilon} \dot{W}_{h} .
$$

where $\dot{W}_{h}$ is defined as in section 3.1. The truncated action functional is then defined as

$$
S_{T}\left(\boldsymbol{u}_{h}\right)=\frac{1}{2} \int_{0}^{T}\left\|\partial_{t} \boldsymbol{u}_{h}+\mathcal{P}_{h}^{\mathrm{H}} \mathcal{G}\left(\boldsymbol{u}_{h}\right)\right\|_{\mathcal{Q}_{h}^{-1}}^{2} d t .
$$

Proposition 4. For any $\boldsymbol{u}$ and $\boldsymbol{v} \in H_{h}$, we have

$$
\left\langle\mathcal{P}_{h}^{\mathrm{H}} \boldsymbol{u}, \boldsymbol{v}\right\rangle_{D}=\langle\boldsymbol{u}, \boldsymbol{v}\rangle_{D}
$$

Proof. We have $\mathcal{P}_{h}^{\mathrm{H}} \boldsymbol{u}=\mathcal{P}_{h} \Pi \boldsymbol{u}$ and

$$
\left\langle\mathcal{P}_{h} \Pi \boldsymbol{u}, \boldsymbol{v}\right\rangle_{D}=\langle\Pi \boldsymbol{u}, \boldsymbol{v}\rangle_{D}=\langle\boldsymbol{u}, \boldsymbol{v}\rangle_{D} .
$$

The first equality is due to the definition of $\mathcal{P}_{h}$. The second equality is due to the Helmholtz-Hodge decomposition, i.e., $(\mathcal{I}-\Pi) \boldsymbol{u} \in H^{\perp}$.

Remark 5. The Helmholtz-Hodge decomposition lays the basis for a family of numerical schemes, called projection methods, to approximate the deterministic Navier-Stokes equaitons. Projection methods are widely used to simulate incompressible flows, and many variants can be found in literature, see the review article [21].

\subsection{Dynamic solver and $M A M$ defined on $V_{h}$ and $H_{h}$}

Depending on how to choose the approximation space and how to implement the Helmholtz-Hodge decomposition, there exist many versions of projection method. Due to our interest of MAM for nonlinear instability of wall-bounded parallel shear flows, we will consider simple geometries and spectral methods for spatial discretization, where a direct construction of divergence-free spaces $V_{h}$ and $H_{h}$ is possible [37].

Since periodic conditions are imposed in $x$ direction, we consider the following truncated Fourier expansion for $\boldsymbol{v} \in V_{h}:=\left(E_{N} \otimes P_{M}\right) \cap V$

$$
\boldsymbol{v}(x, y)=\sum_{|k| \leq N} \boldsymbol{v}_{k}(y) \exp \left(\mathbf{i} 2 \pi k L^{-1} x\right),
$$

with

$$
\boldsymbol{v}_{k}(y)=\sum_{m=0}^{M} \alpha_{k m} \boldsymbol{v}_{k m}^{V}(y)
$$

where $\alpha_{k m}$ are unknown coefficients, $E_{N}$ the set of Fourier modes in $x$ with degree up to $N$, and $P_{M}$ the set of polynomials in $y$ with degree up to $M$. The definition of $V_{h}$ yields the following requirements: for $|k| \leq N, m=0,1, \ldots, M$,

$$
\left\{\begin{aligned}
\nabla \cdot\left(\boldsymbol{v}_{k m}^{V}(y) e^{\mathbf{i} 2 \pi k x / L}\right) & =0 \\
\boldsymbol{v}_{k m}^{V}( \pm 1) & =0 .
\end{aligned}\right.
$$


Let $\boldsymbol{v}_{k m}^{V}(y)=\left[v_{k m, 1}^{V}(y), v_{k m, 2}^{V}(y)\right]^{\top}$. We have

$$
\left\{\begin{aligned}
\left(\mathbf{i} 2 \pi k L^{-1} v_{k m, 1}^{V}(y)+\left(v_{k m, 2}^{V}\right)^{\prime}(y)\right) e^{\mathbf{i} 2 \pi k x / L} & =0, \\
v_{k m, 1}^{V}( \pm 1)=0, \quad v_{k m, 2}^{V}( \pm 1) & =0 .
\end{aligned}\right.
$$

Proposition 5. The basis functions of $V_{h}$ can be defined as follows: For $k \neq 0$,

$$
\boldsymbol{v}_{k m}^{V}(y)=\left(\begin{array}{c}
L_{m}-L_{m+2} \\
-\frac{\mathbf{i} 2 \pi k}{L}\left(\frac{L_{m+1}-L_{m-1}}{2 m+1}-\frac{L_{m+3}-L_{m+1}}{2 m+5}\right)
\end{array}\right), \quad m \geq 1 ;
$$

For $k=0$,

$$
\boldsymbol{v}_{k m}^{V}(y)=\left(\begin{array}{c}
L_{m}-L_{m+2} \\
0
\end{array}\right), \quad m \geq 0,
$$

where $L_{m}(y)$ indicates Legendre polynomials of degree $m$.

Proof. We first look at $k \neq 0$ and $m \geq 1$. Note that

$$
L_{m}( \pm 1)=( \pm 1)^{m} \text {. }
$$

We can take

$$
v_{k m, 1}^{V}(y)=L_{m}(y)-L_{m+2}(y) .
$$

such that $v_{k m, 1}^{V}( \pm 1)=0$. We then have

$$
v_{k m, 2}^{V}(y)=-\frac{\mathbf{i} 2 \pi k}{L} \int_{-1}^{y} v_{k m, 1}^{V}(z) d z=-\frac{\mathbf{i} 2 \pi k}{L} \int_{-1}^{y}\left(L_{m}(z)-L_{m+2}(z)\right) d z,
$$

where the homogeneous boundary conditions are automatically satisfied for $m \geq 1$ due to the orthogonality of Legendre polynomials. Using the derivative recurrence relation:

$$
(2 m+1) L_{m}(y)=L_{m+1}^{\prime}(y)-L_{m-1}^{\prime}(y), \quad m \geq 1,
$$

we have the explicit form of $v_{k m, 2}^{V}(y)$ given in equation (38). For $m=0$, we have

$$
v_{k 0,2}^{V}(y)=-\frac{\mathbf{i} 2 \pi k}{L} \int_{-1}^{y}\left(L_{0}(z)-L_{2}(z)\right) d z+C,
$$

where $C$ is a general constant. Then $v_{k 0,2}^{V}(-1)=0$ implies that $C=0$. However, $v_{k 0,2}^{V}(1)=-\mathbf{i} 4 \pi k L^{-1}$, which is impossible for $k \neq 0$. Thus $m \neq 0$ when $k \neq 0$. When $k=0$, the divergence-free condition yields that $\left(v_{k m, 2}^{V}\right)^{\prime}(y)=0$. Thus $v_{k m, 2}^{V}(y)=0$ due to the homogeneous boundary conditions.

Remark 6. It is noticed that

$$
\overline{\boldsymbol{v}_{k m}^{V}(y) e^{\mathbf{i} 2 \pi k x / L}}=\boldsymbol{v}_{(-k) m}^{V}(y) e^{-\mathbf{i} 2 \pi k x / L},
$$

which means that the following modes

$\boldsymbol{v}_{k m}^{V}(y) e^{\mathbf{i} 2 \pi k x / L}+\boldsymbol{v}_{(-k) m}^{V}(y) e^{-\mathbf{i} 2 \pi k x / L},-\mathbf{i}\left(\boldsymbol{v}_{k m}^{V}(y) e^{\mathbf{i} 2 \pi k x / L}-\boldsymbol{v}_{(-k) m}^{V}(y) e^{-\mathbf{i} 2 \pi k x / L}\right)$ provide a real-valued divergence-free basis. 
For the definition of $H_{h}$ we consider a similar Fourier expansion as equation (36), which yields that

$$
\left\{\begin{aligned}
\nabla \cdot\left(\boldsymbol{v}_{k m}^{H}(y) e^{\mathbf{i} 2 \pi k x / L}\right) & =0 \\
\left.\boldsymbol{v}_{k m}^{H} \cdot \boldsymbol{n}\right|_{y= \pm 1} & =0
\end{aligned}\right.
$$

Let $\boldsymbol{v}_{k m}^{H}(y)=\left[v_{k m, 1}^{H}(y), v_{k m, 2}^{H}(y)\right]^{\top}$. We have

$$
\left\{\begin{aligned}
\left(\mathbf{i} 2 \pi k L^{-1} v_{k m, 1}^{H}(y)+\left(v_{k m, 2}^{H}\right)^{\prime}(y)\right) e^{\mathbf{i} 2 \pi k x / L} & =0, \\
v_{k m, 2}^{H}( \pm 1) & =0 .
\end{aligned}\right.
$$

Proposition 6. The basis functions of $H_{h}$ can be defined as follows: For $k \neq 0$,

$$
\boldsymbol{v}_{k m}^{H}(y)=\left(\begin{array}{c}
L_{m} \\
-\frac{\mathbf{i} 2 \pi k L^{-1}}{2 m+1}\left(L_{m+1}-L_{m-1}\right)
\end{array}\right), \quad m \geq 1 ;
$$

For $k=0$,

$$
\boldsymbol{v}_{k m}^{H}(y)=\left(\begin{array}{c}
L_{m} \\
0
\end{array}\right), \quad m \geq 0,
$$

Proof. The proof is similar to that of Proposition 5 and skipped.

We can then define the approximation space $V_{h}$ as

$$
V_{h}=\operatorname{span}\left\{\phi_{i(k, m)}(\boldsymbol{x})=\boldsymbol{v}_{k m}^{V}(y) e^{\mathbf{i} 2 \pi k x L^{-1}}\right\},
$$

where $|k| \leq N, m=0, \ldots, M$, and $i(k, m)$ indicates a global index of the basis function $\phi_{i(k, m)}$. The meaningful combinations of $k$ and $m$ are given by Proposition 5. It is easy to see that $V_{h} \nsubseteq H_{h}$ if we truncate $H_{h}$ in the same way as $V_{h}$. However, we expect that $V_{h} \subseteq H_{h}$, which mimics numerically the property that $V \subset H$. Furthermore, the cardinality of $V_{h}$ should be the same as that of $H_{h}$ for the Galerkin projection. This implies that we need to take $H_{h}=V_{h}$.

For both the dynamic solver and the MAM, we seek an approximation $\boldsymbol{u}_{h} \in$ $V_{h}$ for any $t$. For the dynamic solver, the temporal discretization will be based on the stiffly-stable scheme, which is a semi-implicit scheme:

$$
\frac{\gamma_{0} \boldsymbol{u}_{h}^{n+1}-\sum_{q=0}^{J_{i}-1} \alpha_{q} \boldsymbol{u}_{h}^{n-q}}{\Delta t}=-\sum_{q=0}^{J_{e}-1} \beta_{q} \mathcal{P}_{h}^{\mathrm{H}}\left(\left(\boldsymbol{u}_{\mathrm{tot}} \cdot \nabla\right) \boldsymbol{u}_{\mathrm{tot}}\right)^{n-q}+\frac{1}{R e} \mathcal{P}_{h}^{\mathrm{H}} \Delta \boldsymbol{u}_{h}^{n+1},
$$

where $J_{i}$ and $J_{e}$ are integration orders for the diffusion term and advection term, respectively, and the coefficients $\gamma_{0}, \alpha_{q}$ and $\beta_{q}$ are determined by the accuracy order of the numerical scheme [31]. The convection term is integrated explicitly and the diffusion term implicitly. The implicit part of equation (44) takes a from

$$
-\mathcal{P}_{h}^{\mathrm{H}} \Delta \boldsymbol{u}_{h}^{n+1}+\lambda \boldsymbol{u}_{h}^{n+1}=\boldsymbol{f},
$$


where $\boldsymbol{f}$ includes all information from current and previous time steps and $\lambda=\gamma_{0} R e / \Delta t$. We then have the Galerkin projection of the above equation as

$$
-\left\langle\mathcal{P}_{h}^{\mathrm{H}} \Delta \boldsymbol{u}_{h}^{n+1}, \boldsymbol{v}_{h}\right\rangle+\lambda\left\langle\boldsymbol{u}_{h}^{n+1}, \boldsymbol{v}_{h}\right\rangle=\left\langle\boldsymbol{f}, \boldsymbol{v}_{h}\right\rangle, \quad \forall \boldsymbol{v}_{h} \in V_{h},
$$

which is equivalent to

$$
\left\langle\nabla \boldsymbol{u}_{h}^{n+1}, \nabla \boldsymbol{v}_{h}\right\rangle+\lambda\left\langle\boldsymbol{u}_{h}^{n+1}, \boldsymbol{v}_{h}\right\rangle=\left\langle\boldsymbol{f}, \boldsymbol{v}_{h}\right\rangle,
$$

due to Proposition 4 and integration by parts. This equation can then be further decomposed according to each Fourier mode in $x$ direction.

For the MAM, we will employ tMAM for the temporal discretization. We define a finite element approximation space for a partitioning of $s \in \Gamma=[0,1]=$ $\cup_{l=0}^{N_{e}} e_{l}=\cup_{l=0}^{N_{e}}\left[s_{l}, s_{l+1}\right]$ :

$$
\begin{aligned}
R_{h} & =\left\{v \in H^{1}([0,1]):\left.v\right|_{e l} \text { is a polynomial of degree } p_{l}\right\}, \\
R_{0, h} & =\left\{v \in R_{h}: v(0)=v(1)=0\right\} .
\end{aligned}
$$

We construct the space-time approximation space for transition paths:

$$
R_{h} \hat{\otimes} V_{h}=\left\{\boldsymbol{u}_{h} \in R_{h} \otimes V_{h}: \boldsymbol{u}_{h}(0)=\boldsymbol{u}_{h, 1}(\boldsymbol{x}), \boldsymbol{u}_{h}(1)=\boldsymbol{u}_{h, 2}(\boldsymbol{x})\right\},
$$

where $\boldsymbol{u}_{h, 1}$ and $\boldsymbol{u}_{h, 2}$ are two states given by the dynamics solver (44). We then seek $\boldsymbol{u}_{h}^{*} \in R_{h} \hat{\otimes} V_{h}$, such that [50]

$$
S_{\hat{T}\left(\boldsymbol{u}_{h}^{*}\right)}\left(\boldsymbol{u}_{h}^{*}\right)=\min _{\substack{\boldsymbol{u}_{h}(0)=\boldsymbol{u}_{h, 1}, \boldsymbol{u}_{h}(1)=\boldsymbol{u}_{h, 2}}} \frac{\hat{T}\left(\boldsymbol{u}_{h}\right)}{2} \int_{0}^{1}\left\|\hat{T}^{-1}\left(\boldsymbol{u}_{h}\right) \partial_{s} \boldsymbol{u}_{h}+\mathcal{P}_{h}^{\mathrm{H}} \mathcal{G}\left(\boldsymbol{u}_{h}\right)\right\|_{\mathcal{Q}_{h}^{-1}}^{2} d s,
$$

where

$$
\hat{T}\left(\boldsymbol{u}_{h}\right)=\left(\frac{\int_{0}^{1}\left\|\partial_{s} \boldsymbol{u}_{h}\right\|_{\mathcal{Q}_{h}^{-1}}^{2} d s}{\int_{0}^{1}\left\|\mathcal{P}_{h}^{\mathrm{H}} \mathcal{G}\left(\boldsymbol{u}_{h}\right)\right\|_{\mathcal{Q}_{h}^{-1}}^{2} d s}\right)^{1 / 2} .
$$

As we have discussed in section 3.2, problem (47) is always well-posed in the sense that $\hat{T}\left(\boldsymbol{u}_{h}^{*}\right)<\infty$ even when the true optimal integral time is $\infty$. In section 4.4 , we provide an $h p$-adaptivity strategy to effectively generate a sequence of refined meshes, which results in a minimizing sequence for problem (47).

We linearize $\mathcal{G}\left(\boldsymbol{u}_{h}\right)$ to obtain the perturbation operator:

$$
\hat{\mathcal{G}}_{\boldsymbol{u}_{h}}^{(1)}\left(\delta \boldsymbol{v}_{h}\right)=\left(\boldsymbol{u}_{\text {tot }} \cdot \nabla\right) \delta \boldsymbol{v}_{h}+\left(\delta \boldsymbol{v}_{h} \cdot \nabla\right) \boldsymbol{u}_{\text {tot }}-R e^{-1} \Delta \delta \boldsymbol{v}_{h},
$$

where the perturbation function $\delta \boldsymbol{v}_{h} \in R_{0, h} \otimes V_{h}$. The first-order variation of $S_{\hat{T}}\left(\boldsymbol{u}_{h}\right)$ can be obtained as

$$
\delta S_{\hat{T}}\left(\delta \boldsymbol{v}_{h}\right)=\hat{T}\left\langle\left(\hat{T}^{-1} \partial_{s}+\mathcal{P}_{h}^{\mathrm{H}} \mathcal{G}\right)\left(\boldsymbol{u}_{h}\right),\left(\hat{T}^{-1} \partial_{s}+\mathcal{P}_{h}^{\mathrm{H}} \hat{\mathcal{G}}_{\boldsymbol{u}_{h}}^{(1)}\right)\left(\delta \boldsymbol{v}_{h}\right)\right\rangle_{\mathcal{Q}_{h}^{-1}, D, s},
$$


where $\langle\cdot, \cdot\rangle_{\mathcal{Q}_{h}^{-1}, D, s}$ indicates the inner product with respect to both $\boldsymbol{x}$ and $s$ weighted by $\mathcal{Q}_{h}^{-1}$. Consider the numerical transition path $\boldsymbol{u}_{h} \in R_{h} \hat{\otimes} V_{h}$ :

$$
\boldsymbol{u}_{h}=\sum_{i=1}^{N_{s}} \sum_{j=1}^{N_{\boldsymbol{x}}} u_{i j} \theta_{i}(s) \phi_{j}(\boldsymbol{x})
$$

where we assume that $R_{h}=\operatorname{span}\left\{\theta_{k}\right\}_{k=1}^{N_{s}}$. The gradient of action functional can be computed as

$$
\frac{\partial S_{\hat{T}}}{\partial u_{i j}}=\left.\delta S_{\hat{T}}\right|_{\delta \boldsymbol{v}_{h}=\theta_{i}(s) \phi_{j}(\boldsymbol{x})}
$$

Remark 7. In [49] we constructed a MAM for 2D Navier-Stokes equations using primitive variables $\boldsymbol{u}$ and $p$, where we considered $\mathcal{G}\left(\boldsymbol{u}_{h}\right)+\nabla p$ instead of $\mathcal{P}_{h}^{\mathrm{H}} \mathcal{G}\left(\boldsymbol{u}_{h}\right)$ in the action functional. In the current formulation, the pressure is removed from the action functional by the Helmholtz-Hodge decomposition. Once the MAP $\boldsymbol{u}_{h}^{*}$ is obtained, the corresponding pressure can be recovered by the Helmholtz-Hodge decompositions since $\nabla p=\left(\mathcal{I}-\mathcal{P}_{h}^{\mathrm{H}}\right) \mathcal{G}\left(\boldsymbol{u}_{h}\right)$.

Remark 8. The incompressibility condition corresponds to a linear constraint of problem (47). Since we restrict $\boldsymbol{u}_{h}$ to the divergence-free space $V_{h}$, the optimization problem becomes an unconstrained one. To this end, a gradienttype optimization solver can be employed to minimize the action functional. In this work, we use the nonlinear Conjugate Gradient method [22, 49].

\subsection{Preconditioning for $M A M$}

Since $\mathcal{Q}_{h}^{-1}$ is self-adjoint, the Euler-Lagrange equation induced by the action functional (47) can be written as

$$
\left(\hat{T}^{-1} \partial_{s}+\mathcal{P}_{h}^{\mathrm{H}} \hat{\mathcal{G}}_{\boldsymbol{u}_{h}}^{(1)}\right)^{*} \mathcal{Q}_{h}^{-1}\left(\hat{T}^{-1} \partial_{s}+\mathcal{P}_{h}^{\mathrm{H}} \mathcal{G}\right)\left(\boldsymbol{u}_{h}\right)=0
$$

where the superscript * indicates the adjoint operator. The Galerkin projection method to solve equation (51) reads: Find $\boldsymbol{u}_{h} \in R_{h} \hat{\otimes} V_{h}$, such that for any $\boldsymbol{v}_{h} \in R_{0, h} \otimes V_{h}$

$$
\left\langle\left(\hat{T}^{-1} \partial_{s}+\mathcal{P}_{h}^{\mathrm{H}} \mathcal{G}\right)\left(\boldsymbol{u}_{h}\right),\left(\hat{T}^{-1} \partial_{s}+\mathcal{P}_{h}^{\mathrm{H}} \hat{\mathcal{G}}_{\boldsymbol{u}_{h}}^{(1)}\right)\left(\boldsymbol{v}_{h}\right)\right\rangle_{\mathcal{Q}_{h}^{-1}, D, s}=0 .
$$

This is consistent with the fact that $\delta S_{\hat{T}} / \delta \boldsymbol{u}_{h}=0$, see equation (49). Equation (52) is a system of nonlinear algebraic equations, which in principle can be solved by the Newton's method. We now look into the Jacobian matrix of system (52). For clarity, we consider the first-order variation of $\delta S_{\hat{T}}\left(\delta \boldsymbol{v}_{h}\right)$ perturbed by $\delta \boldsymbol{w}_{h}$, 
which yields:

$$
\begin{aligned}
& \hat{T}^{-1} \delta\left(\delta S_{\hat{T}}\left(\delta \boldsymbol{v}_{h}\right)\right)\left(\delta \boldsymbol{w}_{h}\right) \\
= & \left\langle\left(\hat{T}^{-1} \partial_{s}+\mathcal{P}_{h}^{\mathrm{H}} \hat{\mathcal{G}}_{\boldsymbol{u}_{h}}^{(1)}\right)\left(\delta \boldsymbol{w}_{h}\right),\left(\hat{T}^{-1} \partial_{s}+\mathcal{P}_{h}^{\mathrm{H}} \hat{\mathcal{G}}_{\boldsymbol{u}_{h}}^{(1)}\right)\left(\delta \boldsymbol{v}_{h}\right)\right\rangle_{\mathcal{Q}_{h}^{-1}, D, s} \\
& +\left\langle\left(\hat{T}^{-1} \partial_{s}+\mathcal{P}_{h}^{\mathrm{H}} \mathcal{G}\right)\left(\boldsymbol{u}_{h}\right),-\hat{T}^{-2} \delta \hat{T} \partial_{s} \delta \boldsymbol{v}_{h}+\mathcal{P}_{h}^{\mathrm{H}} \hat{\mathcal{G}}_{\boldsymbol{u}_{h}}^{(2)}\left(\delta \boldsymbol{v}_{h}, \delta \boldsymbol{w}_{h}\right)\right\rangle_{\mathcal{Q}_{h}^{-1}, D, s} \\
& +\left\langle-\hat{T}^{-2} \delta \hat{T} \partial_{s} \boldsymbol{u}_{h},\left(\hat{T}^{-1} \partial_{s}+\mathcal{P}_{h}^{\mathrm{H}} \hat{\mathcal{G}}_{\boldsymbol{u}_{h}}^{(1)}\right)\left(\delta \boldsymbol{v}_{h}\right)\right\rangle_{\mathcal{Q}_{h}^{-1}, D, s},
\end{aligned}
$$

where $\delta \hat{T}$ is the first-order variation of $\hat{T}$ induced by $\delta \boldsymbol{w}_{h}$ and

$$
\hat{\mathcal{G}}_{\boldsymbol{u}_{h}}^{(2)}\left(\delta \boldsymbol{v}_{h}, \delta \boldsymbol{w}_{h}\right)=\left(\delta \boldsymbol{w}_{h} \cdot \nabla\right) \delta \boldsymbol{v}_{h}+\left(\delta \boldsymbol{v}_{h} \cdot \nabla\right) \delta \boldsymbol{w}_{h} .
$$

Then the Jacobian matrix can be written as

$$
\frac{\partial^{2} S_{\hat{T}}}{\partial u_{i j} \partial u_{i^{\prime} j^{\prime}}}=\left.\delta\left(\delta S_{\hat{T}}\right)\right|_{\delta \boldsymbol{v}_{h}=\theta_{i}(s) \phi_{j}(\boldsymbol{x}), \delta \boldsymbol{w}_{h}=\theta_{i^{\prime}}(s) \phi_{j^{\prime}}(\boldsymbol{x})} .
$$

Due to the existence of $\hat{T}$, which is defined globally, all terms in equation (53) related $\delta T$ are nonzero for any pair of basis functions, which makes the Jacobian matrix full and dense. Thus, a direct application of the Newton's method is not practical. We then try to extract some information from the Jacobian matrix to construct a preconditioner. We first simplify the Jacobian matrix by neglecting the variation of $\hat{T}$ at each iteration step, i.e., $\delta \hat{T}=0$, and the term related to $\mathcal{P}_{h}^{\mathrm{H}} \hat{\mathcal{G}}_{\boldsymbol{u}_{h}}^{(2)}$. We then obtain a positive definite matrix, corresponding to the following bilinear form

$$
\begin{aligned}
& a(\delta \boldsymbol{w}, \delta \boldsymbol{v}) \\
& =\left\langle\left(T^{-1} \partial_{s}+\mathcal{P}_{h}^{\mathrm{H}} \hat{\mathcal{G}}_{\boldsymbol{u}_{h}}^{(1)}\right)\left(\delta \boldsymbol{w}_{h}\right),\left(T^{-1} \partial_{s}+\mathcal{P}_{h}^{\mathrm{H}} \hat{\mathcal{G}}_{\boldsymbol{u}_{h}}^{(1)}\right)\left(\delta \boldsymbol{v}_{h}\right)\right\rangle_{\mathcal{Q}_{h}^{-1}, D, s},
\end{aligned}
$$

where $\hat{T}$ is fixed as $\hat{T}=T$.

Proposition 7. For any $\boldsymbol{u}_{h} \in R_{h} \hat{\otimes} V_{h}$, the matrix $\mathbf{W}: w_{k, k^{\prime}}=a\left(\Phi_{k(i, j)}, \Phi_{k^{\prime}\left(i^{\prime}, j^{\prime}\right)}\right)$ is symmetric and positive definite, where $\Phi_{k(i, j)}=\theta_{i}(s) \phi_{j}(\boldsymbol{x})$ and $k(i, j)$ indicates a global index of the basis function $\theta_{i}(s) \phi_{j}(\boldsymbol{x})$.

Proof. If is easy to see that $a(\boldsymbol{v}, \boldsymbol{w})$ is symmetric and $a(\boldsymbol{v}, \boldsymbol{v}) \geq 0$ for any $\boldsymbol{v}, \boldsymbol{w} \in R_{0, h} \otimes V_{h}$. We now verify that $a(\boldsymbol{v}, \boldsymbol{v})=0$ only has a trivial solution. Since the inner product $\langle\cdot, \cdot\rangle_{\mathcal{Q}_{h}^{-1}, D, s}$ defines a weighted $L_{2}$ norm, $a(\boldsymbol{v}, \boldsymbol{v})=0$ implies that

$$
T^{-1} \partial_{s} \boldsymbol{v}+\mathcal{P}_{h}^{\mathrm{H}} \hat{\mathcal{G}}_{\boldsymbol{u}_{h}}^{(1)}(\boldsymbol{v})=0 .
$$

Let $\left\{\gamma_{i}(\boldsymbol{x})\right\}_{i=1}^{N_{x}}$ be an orthonormal basis of $H_{h}=V_{h}$. Let $\boldsymbol{v}=\sum_{i} v_{i}(s) \gamma_{i}(\boldsymbol{x})$. We have for any $\gamma_{j}(\boldsymbol{x})$

$$
\begin{aligned}
& \left\langle\partial_{s} \boldsymbol{v}, \gamma_{j}\right\rangle_{D}+T\left\langle\mathcal{P}_{h}^{\mathrm{H}} \hat{\mathcal{G}}_{\boldsymbol{u}_{h}}^{(1)}(\boldsymbol{v}), \gamma_{j}\right\rangle_{D}=\frac{d v_{j}}{d s}+T\left\langle\hat{\mathcal{G}}_{\boldsymbol{u}_{h}}^{(1)}(\boldsymbol{v}), \gamma_{j}\right\rangle_{D} \\
= & \left.\frac{d v_{j}}{d s}+T \sum_{i} v_{i}\left(\left\langle\boldsymbol{u}_{\mathrm{tot}} \cdot \nabla\right) \gamma_{i}, \gamma_{j}\right\rangle_{D}+\left\langle\left(\gamma_{i} \cdot \nabla\right) \boldsymbol{u}_{h}, \gamma_{j}\right\rangle_{D}-R e^{-1}\left\langle\Delta \gamma_{i}, \gamma_{j}\right\rangle_{D}\right)=0,
\end{aligned}
$$


which is a linear first-order system with respect to $s$. Due to the uniqueness theorem and the fact that $v_{j}(0)=0, \forall j$, we have $v_{j}(s) \equiv 0$, i.e., $\boldsymbol{v}=0$. This concludes the proof.

With respect to the matrix $\mathbf{W}$, we have the following options for preconditioning:

(1). Use $\mathbf{W}^{-1}$ as the preconditioner. One computational issue is that $\mathbf{W}$ depends on current $\boldsymbol{u}_{h}$, which is also dense within each time element due to the nonlinearity of the advection term.

(2). Use the diagonal entries of $\mathbf{W}$ to construct a diagonal preconditioner. This strategy does not introduce new computational cost since it only uses the information required by the gradient. This preconditioner was implemented in [49] for a MAM of N-S equations subject to a fixed integration time $T$, where a speed-up of $O(10)$ was observed.

(3). Remove the terms in $a(\delta \boldsymbol{w}, \delta \boldsymbol{v})$ related to the advection term, which results in

$$
\hat{a}(\delta \boldsymbol{w}, \delta \boldsymbol{v})=\left\langle\left(T^{-1} \partial_{s}-R e^{-1} \mathcal{P}_{h}^{\mathrm{H}} \Delta\right) \delta \boldsymbol{w},\left(T^{-1} \partial_{s}-R e^{-1} \mathcal{P}_{h}^{H} \Delta\right) \delta \boldsymbol{v}\right\rangle_{\mathcal{Q}_{h}^{-1}, D, s} .
$$

This bilinear form corresponds to the leading terms $\left(-T^{-2} \partial_{s s}+R e^{-2} \Delta^{2}\right) \boldsymbol{u}_{h}$ of the Euler-Lagrange equation. If the integration time $T$ is time independent, the stiffness matrix induced by $\hat{a}(\delta \boldsymbol{w}, \delta \boldsymbol{v})$ only needs to be assembled once. However, at the early stage of convergence, $T\left(\boldsymbol{u}_{h}\right)$ varies significantly, which means that at each iteration step we need to assemble the stiffness matrix. This implies the preconditioner given by $\hat{a}(\delta \boldsymbol{w}, \delta \boldsymbol{v})$ can be expensive.

(4). There exist optimization algorithms, such as L-BFGS, which accelerate the convergence by approximating the Hessian using the curvature information from recent iterations. These techniques may be used as a second-level preconditioner to compensate our simplification of the Jacobian matrix.

In this paper, we would choose option (2) and leave options (3) and (4) for future study.

\subsection{Adaptivity in time direction}

Adaptivity in time direction is necessary for all MAMs formulated with respect to time mainly due to two reasons: 1) The optimal integration time for the action functional to reach the minimum can be infinity, e.g., $\boldsymbol{u}_{h, 1}$ is the laminar state, and 2) Minimizing the action functional corresponds to solving a $(d+1)$-dimensional boundary value problem on $\Gamma \times D$, where the number of unknowns may significantly limit the problem size we are able to study.

In [50], we developed an $h$-type adaptive tMAM, where local refinement subject to a physically based criterion was successfully used to deal with the quasi-potential. More specifically, the $h$-type tMAM can achieve the optimal convergence rate $O\left(h^{2 p}\right)$ in terms of the error of the action functional, where $h$ indicates the element size in time direction and $p$ the polynomial order in each element. To alleviate the limitation of physically based adaptivity, we employed the derivative recovery technique to construct a posteriori error estimate of 
the MAP, which resulted in an $h p$-adaptive tMAM [51]. There exist two key element-wise estimators $\eta_{e_{i}}^{m}$ and $\eta_{e_{i}}^{a}$ for the $h p$-adaptivity in time direction. The estimator $\eta_{e_{i}}^{m}$ measures the error of model approximation, i.e., $T_{h}^{*} \approx \hat{T}\left(\boldsymbol{u}_{h}^{*}\right)$ is always finite due to linear scaling while $T_{h}^{*}$ can be infinite (see equation (25)). The estimator $\eta_{e_{i}}^{a}$ measures the error of path approximation given by the finite element discretization. Associated with $\eta_{e_{i}}^{a}$, a regularity indicator $\alpha_{e_{i}}$ can also be defined to estimate the smoothness of the path in element $e_{i}$. Based on $\eta_{e_{i}}^{m}, h$ type adaptivity is used to deal with the error of model approximation; based on $\eta_{e_{i}}^{a}$ and $\alpha_{e_{i}}, h p$-adaptivity is used to deal with the error of path approximation.

We will adapt the $h p$-adaptive tMAM [51] for the Navier-Stokes equations. The path $\boldsymbol{u}_{h} \in R_{h} \hat{\otimes} V_{h}$ can be regarded as a vector function $\mathbf{u}_{h, s} \in \mathbb{R}^{N_{x} \times N_{y}}$ of $s$, where $N_{x}$ is the number of Fourier modes in $x$ direction and $N_{y}$ the number of modified Legendre modes in $y$ direction. Then $\left\|\boldsymbol{u}_{h}\right\|_{L_{2}(D)}$ is an equivalent norm of $\left|\mathbf{u}_{h, s}\right|_{\ell_{2}}$ for any $s$. In [51], we established a posteriori error estimates with respect to norm $|\cdot|_{\ell_{2}}$ for vector functions. Following is our main strategy for a posteriori error estimates: First, we use the deviation from the arc length constraint to define the estimator $\eta_{e_{i}}^{m}$. The arc length constraint [27]

$$
\left\|\partial_{t} \boldsymbol{u}_{h}\right\|_{L_{2}(D)}=\left\|\mathcal{P}_{h}^{\mathrm{H}} \mathcal{G}\left(\boldsymbol{u}_{h}\right)\right\|_{L_{2}(D)}, \quad \forall t .
$$

is a necessary condition satisfied by the MAP. Due to the discretization and linear time scaling, this constraint cannot be exactly maintained especially when the optimal integration time is $\infty$. We then define

$$
\left(\eta_{e_{i}}^{m}\right)^{2}=\hat{T}\left(\boldsymbol{u}_{h}\right) \int_{s_{i}}^{s_{i+1}}\left(\hat{T}^{-1}\left(\boldsymbol{u}_{h}\right)\left\|\partial_{s} \boldsymbol{u}_{h}\right\|_{L_{2}(D)}-\left\|\mathcal{P}_{h}^{H} \mathcal{G}\left(\boldsymbol{u}_{h}\right)\right\|_{L_{2}(D)}\right)^{2} d s,
$$

using the residual from the arc length constraint. Second, we take advantage of the super-convergence of derivative recovery operator $\mathcal{R}$. For illustration, let us assume that $\mathbf{u}_{h, s}$ is discretized by linear finite elements in time direction, each component of the first-order derivative $\mathbf{u}_{h, s}^{\prime}$ is then piece-wise constant with respect to $s$. The derivative recovery $\mathcal{R} \mathbf{u}_{h, s}^{\prime}$ will project each component of $\mathbf{u}_{h, s}^{\prime}$ onto linear finite element space and then smooth the projection. $\mathcal{R} \mathbf{u}_{h, s}^{\prime}$ is a better approximation of the true solution than $\mathbf{u}_{h, s}^{\prime}$ such that we can define a posteriori error estimate using

$$
\left(\eta_{e_{i}}^{a}\right)^{2}=\int_{0}^{1}\left\|(\mathcal{I}-\mathcal{R}) \partial_{s} \boldsymbol{u}_{h}\right\|_{L_{2}(D)}^{2} d s \sim \int_{0}^{1}\left|(\mathcal{I}-\mathcal{R}) \mathbf{u}_{h, s}^{\prime}\right|_{\ell_{2}}^{2} d s,
$$

where we replace the norm $|\cdot|_{\ell_{2}}$ with $\|\cdot\|_{L_{2}(D)}$. We also consider an extension $\mathcal{E} \mathbf{u}_{h, s}$, which is a quadratic extension of $\mathbf{u}_{h, s}$ obtained by post processing. If the regularity is good, we expect that $\left(\mathcal{E} \mathbf{u}_{h, s}\right)^{\prime}$ is comparable to $\mathcal{R} \mathbf{u}_{h, s}^{\prime}$, otherwise, we use the ratio

$$
\left(\alpha_{e_{i}}\right)^{2}=\frac{\int_{0}^{1}\left\|(\mathcal{I}-\mathcal{R}) \partial_{s} \boldsymbol{u}_{h}\right\|_{L_{2}(D)}^{2} d s .}{\int_{0}^{1}\left\|\partial_{s}\left(\boldsymbol{u}_{h}-\mathcal{E} \boldsymbol{u}_{h}\right)\right\|_{L_{2}(D)}^{2} d s .}
$$

as a measure of the regularity. This procedure can be generalized to an approximation given by $h p$ finite elements. More details about the definition of $\mathcal{R}$ and $\mathcal{E}$ can be found in [51]. 
To this end, we have all the estimators we need and the adaptivity criteria can be borrowed directly from [51]. We here only describe the basic adaptive strategy: After the results are obtained for the current mesh, we compute the three indicators $\eta_{e_{i}}^{m}, \eta_{e_{i}}^{a}$ and $\alpha_{e_{i}}$ elementwisely and the average polynomial order $p_{\text {ave }}$ through all time elements. If the current number of degrees of freedom (DOF) is $M$, we will set up a target number $M\left(1+p_{\text {ave }}\right) / p_{\text {ave }}$ of DOFs for the new time mesh. We will separate the new DOFs into two parts: one part is to deal with the error of path approximation, where we consider $h$-refinement or $p$-refinement based on $\eta_{e_{i}}^{a}$ and $\alpha_{e_{i}}$; the other part is to deal with the error of model approximation, i.e., the deviation from the arc length constraint (56), which will be done by suppressing the error $\eta_{e_{i}}^{m}$ via $h$-refinement. Numerical experiments show that we only need a small portion of the new DOFs for the error of model approximation. A typical choice can be $90 \%$ of the new DOFs

for the error of path approximation and $10 \%$ of the new DOFs for the error of model approximation. We refer to [51] for more details about the adaptivity strategy.

\subsection{Some issues related to numerical efficiency}

There exist several other computational issues that are particularly related to numerical efficiency. First, it is seen from equation (49) that we need to compute $\mathcal{P}_{h}^{\mathrm{H}} \mathcal{G}\left(\boldsymbol{u}_{h}\right)$ and $\mathcal{P}_{h}^{\mathrm{H}} \hat{\mathcal{G}}_{\boldsymbol{u}_{h}}^{(1)}\left(\delta \boldsymbol{v}_{h}\right)$ for each component of $\nabla S_{\hat{T}}\left(\boldsymbol{u}_{h}\right)$. In general, the Helmholtz-Hodge decomposition can be done by solving an elliptic equation: taking the divergence of $\Pi \boldsymbol{u}=\boldsymbol{u}-\nabla q$, we have

$$
\left\{\begin{aligned}
\Delta q & =\nabla \cdot \boldsymbol{u} \\
\frac{\partial q}{\partial \mathbf{n}} & =\left.\boldsymbol{u} \cdot \mathbf{n}\right|_{y= \pm 1},\left.q\right|_{x=0}=\left.q\right|_{x=L}
\end{aligned}\right.
$$

However, this is only necessary when we do not have a divergence-free space for $\Pi \boldsymbol{u}$. From Proposition 4 we have for any $\boldsymbol{v} \in H_{h}$

$$
\left\langle\mathcal{P}_{h}^{\mathrm{H}} \mathcal{G}\left(\boldsymbol{u}_{h}\right), \boldsymbol{v}\right\rangle_{D}=\left\langle\mathcal{G}\left(\boldsymbol{u}_{h}\right), \boldsymbol{v}\right\rangle_{D},\left\langle\mathcal{P}_{h}^{\mathrm{H}} \hat{\mathcal{G}}_{\boldsymbol{u}_{h}}^{(1)}\left(\delta \boldsymbol{v}_{h}\right), \boldsymbol{v}\right\rangle_{D}=\left\langle\hat{\mathcal{G}}_{\boldsymbol{u}_{h}}^{(1)}\left(\delta \boldsymbol{v}_{h}\right), \boldsymbol{v}\right\rangle_{D} .
$$

Then $\mathcal{P}_{h} \Pi \mathcal{G}\left(\boldsymbol{u}_{h}\right)$ and $\mathcal{P}_{h} \Pi \hat{\mathcal{G}}_{\boldsymbol{u}_{h}}^{(1)}\left(\delta \boldsymbol{v}_{h}\right)$ can be obtained by solving the linear system given by the mass matrix induced by the basis functions of $H_{h}=V_{h}$. This can be done efficiently: FFT can be implemented in $x$ direction, and the Cholesky factorization in $y$ direction.

Second, the aliasing error deserves some attention. When we considered a direct discretization of the original action functional (4) in [49], we need to compute inner products like $\left\langle\left(\boldsymbol{u}_{h} \cdot \nabla\right) \boldsymbol{u}_{h},\left(\boldsymbol{v}_{h} \cdot \nabla\right) \boldsymbol{w}_{h}\right\rangle_{\mathcal{Q}_{h}^{-1}, D, s}$ induced by the convection term, where $\boldsymbol{u}_{h}, \boldsymbol{v}_{h}, \boldsymbol{w}_{h} \in V_{h}$. Let $\mathcal{Q}_{h}=\mathcal{I}$ for simplicity. To compute such an inner product exactly, we need $O(2 N)$ Gauss-type quadrature points in each direction, i.e., $x-, y$ - and $s$-direction, where $N$ indicates the polynomial order in a certain direction. So on the subdomain $e_{l} \times D$, the number of unknowns is $O\left(N_{x} N_{y} N_{s}\right)$ while the number of quadrature points should be $O\left(8 N_{x} N_{y} N_{s}\right)$ to avoid aliasing errors, where $N_{*}$ is the polynomial order in the direction indicated by the star. If we consider the action functional given by equation (34), 
this issue can be alleviated. Since $\mathcal{P}_{h}^{\mathrm{H}} \mathcal{G}\left(\boldsymbol{u}_{h}\right), \mathcal{P}_{h}^{\mathrm{H}} \mathcal{G}_{\boldsymbol{u}_{h}}^{(1)}\left(\delta \boldsymbol{v}_{h}\right) \in H_{h}$, the aliasing errors are mainly related to the terms $\left\langle\mathcal{G}\left(\boldsymbol{u}_{h}\right), \boldsymbol{v}\right\rangle_{D}$ and $\left\langle\hat{\mathcal{G}}_{\boldsymbol{u}_{h}}^{(1)}\left(\delta \boldsymbol{v}_{h}\right), \boldsymbol{v}\right\rangle_{D}$ in equation (60). Based on the quadratic nonlinearity of the advection term, there exist some de-aliasing techniques that can be used, e.g., the $3 / 2$ padding rule [39], implicit padding [1], etc.

Third, the pressure can be obtained in a post-processing stage. Due to the divergence-free space, the pressure disappears in the action functional. For twodimensional cases, the number of unknowns is thus reduced by half compared to the MAM in [49]. Furthermore, the divergence-free space can be generalized straightforwardly to three dimensional cases, where we only need to consider two unknown components of the velocity field.

\section{Numerical experiments}

\subsection{An artificial trajectory}

We first test the following artificial trajectory:

$$
u=\left(y^{3}-y\right) \cos \left(\frac{2 \pi k x}{L}\right) e^{t / 2}, \quad v=\frac{\pi k}{L}\left(\frac{y^{4}}{2}-y^{2}+\frac{1}{2}\right) \sin \left(\frac{2 \pi k x}{L}\right) e^{t / 2},
$$

which corresponds to one divergence-free basis mode scaled by $e^{t}$. To make this trajectory an exact solution of the deterministic N-S equations, we need to add a corresponding force term. We then choose $(u(t), v(t))$ at $t=0,6$ as the initial and final states for the MAM. In Figure 1, we plot both $h$ - and $p$-convergence with respect to the number of degrees of freedom (DOF). The errors of the action functional of the approximated MAP are plotted on the left, and the errors of the approximated integration time on the right. The DOF is represented as $m N_{\boldsymbol{x}}$, where $m$ corresponds to different cases of temporal discretization and the spatial discretization remains the same. Uniform temporal meshes are used for the $h$-convergence, and the optimal convergence rate $O\left(h^{2 p}\right)$ is obtained for the error of action functional [50].

\subsection{A trajectory from the dynamic solver}

We take two points on one trajectory given by the dynamic solver as the initial and final state for MAM. More specifically, we let $R e=3500$ and $L=5$, for which the deterministic N-S equations have a traveling wave solution denoted as $U(t)$. The spatial disretization is given by a divergence-free approximation space $\operatorname{span}\left\{\phi_{i(k, m)}(\boldsymbol{x})\right\}$ with $0 \leq|k| \leq 4,0 \leq m \leq 32$, whose cardinality is denoted by $N_{\boldsymbol{x}}$, i.e., $1 \leq i(k, m) \leq N_{\boldsymbol{x}}$. Once the traveling wave solution is fully developed, we pick two snapshots $U\left(t_{0}\right)$ and $U\left(t_{0}+\tau\right)$ with $\tau=6$. According to the definition of action functional, see equation (47), the minimum is reached at

$$
\hat{T}^{-1}\left(\boldsymbol{u}_{h}^{*}\right) \partial_{s} \boldsymbol{u}_{h}^{*}+\mathcal{P}_{h}^{H} \mathcal{G}\left(\boldsymbol{u}_{h}^{*}\right)=0
$$

where $\hat{T}^{-1}\left(\boldsymbol{u}_{h}^{*}\right)=\tau$. In other words, the semi-discrete deterministic N-S equations (32) is solved by $\boldsymbol{u}_{h}^{*}$. 

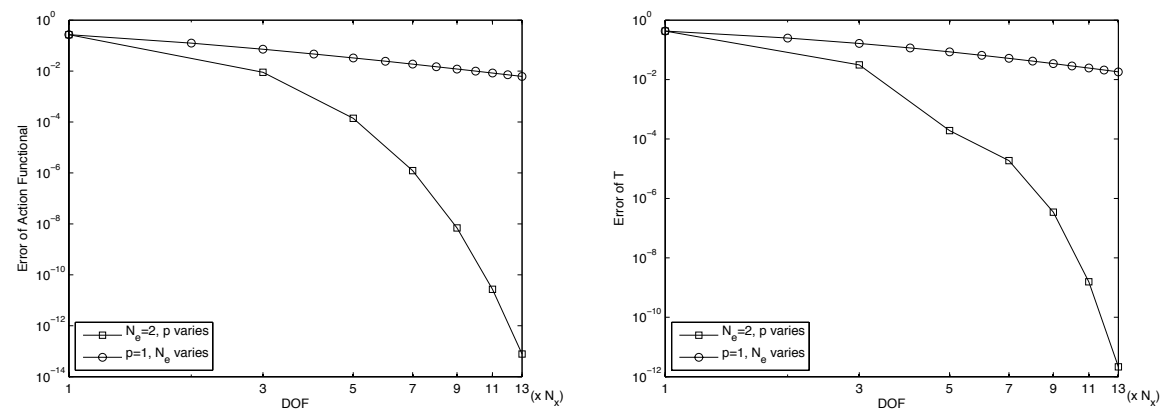

Figure 1: Convergence of tMAM. The initial and final states are located on the trajectory given by equation (61) at $t=0$ and $t=6$. Left: Errors of the action functional of the approximated MAP. Right: Errors of the estimated optimal integration time.

In Figure 2, we present the convergence behavior of tMAM for this case. Note that the approximated trajectory $U(t)$ is located in the following set

$$
\{\phi(t) \mid \rho(\phi(t), \hat{U}(t)) \leq \epsilon\},
$$

where $\hat{U}(t)$ is an exact solution of equation (32), $\epsilon$ is determined by the accuracy of time integration of the dynamic solver, and $\rho(\phi, \hat{U})=\max _{t_{0} \leq t \leq t_{0}+\tau} \| \phi(t)-$ $\hat{U}(t) \|_{L_{2}(D)}$. Then along the trajectory $U(t)$, we have $S_{T}(U) \leq \frac{\tau}{2} \epsilon^{2}$. Although $U\left(t_{0}\right)$ and $U\left(t_{0}+\tau\right)$ are not exactly located on a true solution of equation (32), we at least have $S_{T}\left(\boldsymbol{u}_{h}^{*}\right) \leq \frac{\tau}{2} \epsilon^{2}$ if the temporal resolution of $\boldsymbol{u}_{h}^{*}$ is good enough. We then compute $U(t)$ using a second-order dynamic solver with time step size $10^{-4}$. It is seen in Figure 2 that the error of action functional, i.e., $S_{T}\left(\boldsymbol{u}_{h}^{*}\right)$ decays exponentially to zero as the polynomial order $p$ increases. In other words, we do not have the discrepancy between the dynamic solver and the MAM from discretizing operators $\mathcal{P}_{h}^{\mathrm{H}} \mathcal{G}$ and $\mathcal{G}$ (see section 3 and [49]). The $h$-convergence is based on uniform temporal discretization and the optimal rate $O\left(h^{2 p}\right)$ is observed with respect to the error of action functional. We also observe that the error of the approximated integration time stops to decay when the polynomial order is large enough. This is because $U\left(t_{0}\right)$ and $U\left(t_{0}+\tau\right)$ are located on a numerical trajectory instead of the exact one.

In Figure 3, we examine the effectiveness of the diagonal preconditioner for tMAM. First, the diagonal preconditioner becomes more effective as the error decays. This is because we remove $\delta \hat{T}$ in the bilinear form (55), which can be relatively big at the early stage. Second, the speedup appears to be larger when the polynomial order is higher. The best speedup we can obtain is $O(10)$.

\section{3. hp-adaptivity}

We here demonstrate the $h p$-adaptivity of the tMAM for N-S equations. The initial and final states are the same as those in the previous section. We start with a mesh that has two linear elements, and then let the mesh evolve with 
respect to $h p$-adaptivity. The convergence behavior is given in Figure 4 . We see that an overall trend of exponential convergence is obtained with respect to DOFs. Compared to the left plot in Figure 2, the $h p$-convergence is less efficient than the $p$-convergence. This is expected because the initial and finite states are from a trajectory subject to a finite integration time. The second reason is that the mesh is really coarse at the early stage where the $h$-refinement is more preferred to the $p$-refinement. The third reason is that we use $h$-refinement to deal with error of model approximation due to the consideration of robustness. The final mesh has 9 temporal elements with a distribution of polynomial order as $6,6,6,6,6,5,5,8,8$.
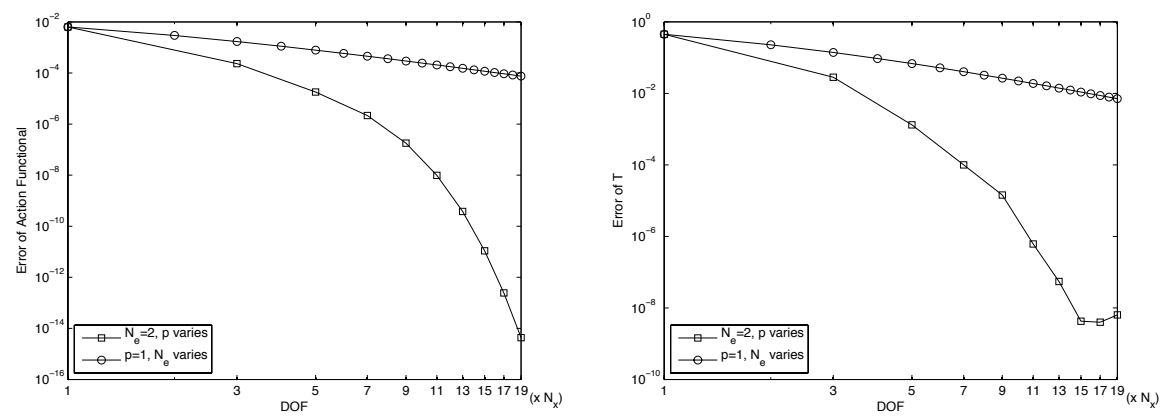

Figure 2: Convergence of tMAM. The initial and final states are located on a traveling wave trajectory given by the dynamic solver. Left: Errors of the action functional of the approximated MAP. Right: Errors of the estimated optimal integration time.
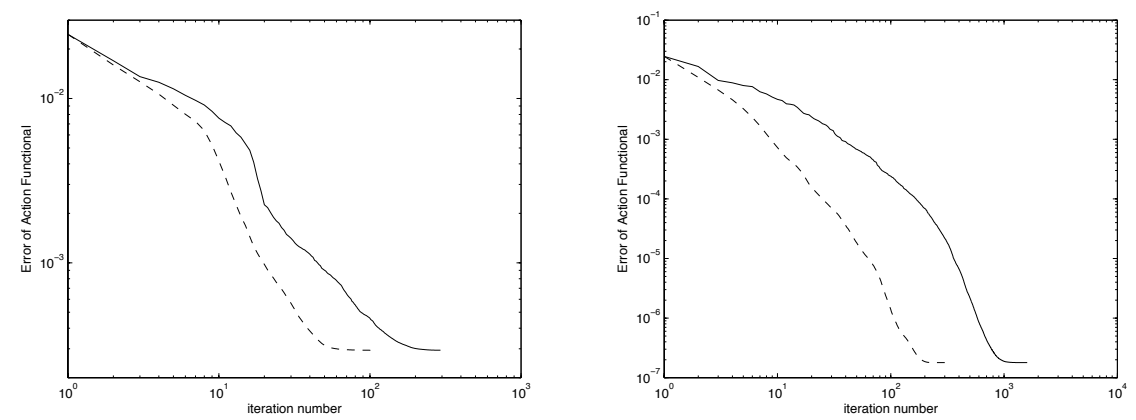

Figure 3: Effectiveness of the diagonal preconditioner for tMAM. The solid line is given by nonlinear CG without preconditioner, and the dash line is given by the same solver with the diagonal preconditioner. Left: $N_{e}=10, p=1$. Right: $N_{e}=2, p=5$.

\section{Summary and discussions}

In this paper we discussed the relation between dynamic solver and minimum action method, based on which we developed a new minimum action method 


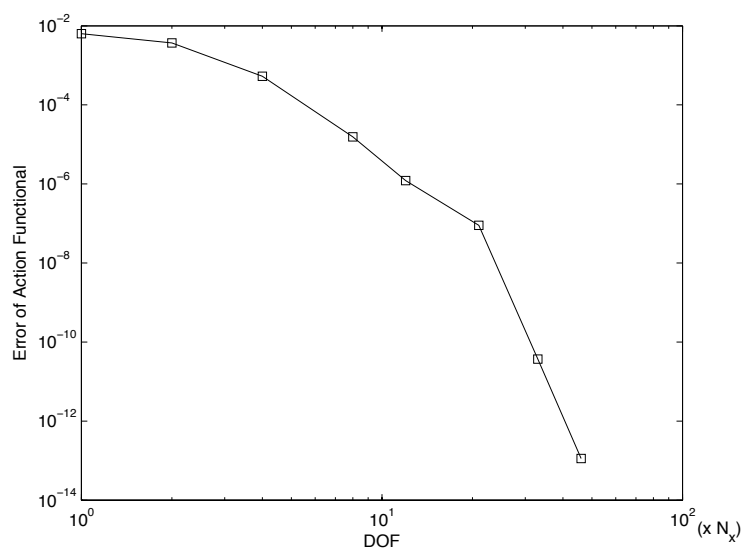

Figure 4: Convergence behavior of $h p$-adaptive tMAM starting from $N_{e}=2$ and $p=1$.

for two-dimensional Navier-Stokes equations. The new MAM has several features: First, the spatial discretization is given by a divergence-free space, which eliminates the pressure in both dynamic solver and MAM. The number of degrees of freedom is then significantly reduced. Second, the new MAM is able to deal with quasi-potential with respect to time, which can be used to study the transition between any two points in the phase space. This provides more flexibility to study the phase space of N-S equations. Third, the $h p$-adaptivity of MAM is based on approximation theory instead of physical intuition, which can be more effective to reduce the number of degrees of freedom from the temporal discretization. Fourth, the accumulation of residual errors from spatial discretization is alleviated, which is important for transitions at a large time scale.

From the algorithm point of view, the preconditioning deserves further study. Due to the variation of integration time at each iteration step and the fact that the integration time is estimated by the whole path, the Hessian can become dense, which makes the construction of preconditioner more difficult. We also observed that the diagonal preconditioner is not quite effective at the early stage because we remove the variation of integration time.

\section{Acknowledgement}

X. Wan was supported by AFOSR Grant FA9550-15-1-0051 and NSF Grant DMS-1620026, and H. Yu was supported by NNSFC Grants 11101413, 11371358, and Major Program of NNSFC Grant 91530322.

[1] J. Bowman and M. Roberts, Efficient dealiased convolutions without padding, SIAM J. Sci. Comput., 33(1) (2011), 386-406. 
[2] , Z. Brzezniak, S. Cerrai and M. Fraidlin, Quasipotential and exit time for $2 D$ stochastic Navier-Sokes equations driven by space time white noise, (2014) arXiv:1401.6299.

[3] C. J. Cerjan and W. H. Miller, On finding transition states, J. Chem. Phys. 75(6) (1981), 2800-2806.

[4] C. Canuto, M. Y. Hussaini, A. Quarteroni and T. A. Zang, Spectral Methods in Fluid Dynamics, Springer-Verlag, 1988.

[5] S. Cerrai and M. Freidlin, Approximation of quasi-potential and exit problems for multidimensional RDE's with noise, Trans. Amer. Math. Soc, 363(7) (2011), 3853-3892.

[6] A. J. Chorin, The numerical solution of the Navier-Stokes equations for an incompressible fluid, Bull. Amer. Math. Soc., 73 (1967), 928.

[7] G. Ciccotti, T. Lelievre, and E. Vanden-Eijnden, Projection of diffusions on submanifolds: Application to mean force computation, Comm. Pure Appl. Math., 61 (2008), 371-408.

[8] A. Debussche and J. Printems, Weak order for the discretization of the stochastic heat equation, Math. Comput., 78(266) (2009), 845-863.

[9] Q. Du and L. Zhang, A constrained string method and its numerical analysis, Commun. Math. Sci., 7 (2009), 1039-1051.

[10] W. E, W. Ren and E. Vanden-Eijnden, String method for the study of rare events, Phys. Rev. B, 66 (2002), 052301.

[11] W. E, W. Ren and E. Vanden-Eijnden, Minimum action method for the study of rare events, Commun. Pure Appl. Math., 57 (2004), 637-565.

[12] W. E, W. Ren and E. Vanden-Eijnden, Simplified and improved string method for computing the minimum energy paths in barrier-crossing events, J. Chem. Phys., 126 (2007), 164103.

[13] W. E and X. Zhou, The gentlest ascent dynamics, Nonlinearity, 24 (2011), $1831-1842$.

[14] W. E, X. Zhou and X. Cheng, Subcritical bifurcation in spatially extended systems, Nonlinearity, 25 (2012), 761-779.

[15] W. G. Faris and G. Jona-Lasinio, Large fluctuations for a nonlinear heat equation with noise, J. Phys. A: Math. Gen. 15 (1982), 3025-3055.

[16] M. I. Freidlin and A. D. Wentzell, Random Perturbations of Dynamical Systems, Springer-Verlag, New York, 2nd Edition, 1998.

[17] T. Grafke, T. Schaefer and E. Vanden-Eijnden, Long Term Effects of Small Random Perturbations on Dynamical Systems: Theoretical and Computational Tools, arXiv:1604.03818. 
[18] W. Gao, J. Leng and X. Zhou, An iterative minimization formulation for saddle point search, SIAM J. Number. Anal., 53(4) (2015), 1786-1805.

[19] W. D. George, J. D. Hellums and B. Martin, Finite-amplitude neutral disturbances in plane Poiseuille flow, J. Fluid Mech., 63 (1974), 765-771.

[20] T. Grafke, R. Grauer and T. Schäfer, The instanton method and its numerical implementation in fluid mechanics, J. Phys. A Math. Theor. 48(33) 2015333001.

[21] J. L. Guermond, P. Minev and J. Shen, An overview of projection methods for incompressible flows, Comput. Methods Appl. Mech. Engrg., 195 (2006), 6011-6045.

$[22]$ W. W. Hager and H. Zhang, A new conjugate gradient method with guaranteed descent and an efficient line search, SIAM J. Optim., 16(1) (2005), 170-192.

[23] W. W. Hager and H. Zhang, The limited memory conjugate gradient method, SIAM J. Optim., 23(4) (2013), 2150-2168.

[24] M. Hairer and H. Weber, Large deviations for white-noise driven, nonlinear stochastic PDEs in two and three dimensions, Ann. Fac. Sci. Toulouse Math., 24(6) (2015), 55-92.

[25] E. Hausenblas, Approximation for semilinear stochastic evolution equations, Potential Anal., 18 (2003), 141-186.

[26] G. Henkelman and H. Jónsson, A dimer method for finding saddle points on high dimensional potential surfaces using only first derivatives, J. Chem. Phys., 111 (1999), 7010-7022.

[27] M. Heymann and E. Vanden-Eijnden, The geometric minimum action method: A least action principle on the space of curves, Commun. Pure Appl. Math., 61 (2008), 1052-1117.

[28] A. Jentzen and P. E. Kloeden, The numerical approximation of stochastic partial differential equations, Milan J. Math., 77 (2009), 205-244.

[29] H. Jònsson, G. Mills and K. W. Jacobsen, Nudged elastic band method for finding minimum energy paths of transitions, Classical and Quantum Dynamics in Condensed Phase Simulations, Ed. B. Berne, G. Ciccotti and D. Coker, 1998.

[30] N. G. van Kampen, Stochastic Processes in Physics and Chemistry, NorthHolland, 1981.

[31] G. E. Karniadakis and S. J. Sherwin, Spectral/hp Element Methods for Computational Fluid Dynamics, Oxford University Press, 2nd Edition, 2005. 
[32] M. Kovávs, S. Larsson and F. Lindgren, Strong convergence of the finite element method with truncated noise for semilinear parabolic stochastic equations with additive noise, Numer. Algor., 53 (2010), 309-320.

[33] M. Kovávs, S. Larsson and F. Lindgren, Weak convergence of finite element approximations of linear stochastic evolution equations with additive noise, BIT Numer. Math., 52 (2012), 85-108.

[34] R. Kruse, Optimal error estimates of Galerkin finite element methods for stochastic partial differential equations with multiplicative noise, IMA J. Numer. Anal., 34(1) (2014), 217-251.

[35] A. Monokrousos, A. Bottaro, L. Brandt, A. Di Vita and D. S. Henningson, Nonequilibrium Thermodynamics and the Optimal Path to Turbulence in Shear Flows, Phys. Rev. Letter, 106 (2011), 134502.

[36] T. Li, X. Li and P. Zhang, Finding Transition Pathways on Manifolds, Multiscale Model. Sim., 14(1) (2016), 173-206.

[37] R. D. Moser, P. Moin and A. Leonard, A spectral numerical method for the Navier-Stokes equations with applications to Taylor-Couett flow, J. Comput. Phys., 52 (1983), 52-544.

[38] L. Onsager and S. Machlup, Fluctuations and irreversible processes, Phys. Rev., 91 (1953), 1505-1512.

[39] S. Orszag, Elimination of aliasing in finite-difference schemes by filtering high-wavenumber components, J. Atmos. Sci., 28 (1971), 1074.

[40] S. Orszag and A. Patera, Secondary instability of wall-bounded shear flows, J. Fluid Mech., 128 (1983), 347-385.

[41] W. Ren and E. Vanden-Eijnden, A climbing string method for saddle point search, J. Chem. Phys., 138 (2013), 134105.

[42] T. M. Schneider, J. F. Gibson, M. Lagha, F. De Lillo and B. Eckhardt, Laminar-turbulent boundary in plane Couette flow, Phys. Rev. E, 78 (2008), 037301.

[43] S. S. Sritharan and P. Sundar, Large deviations for the two-dimensional Navier-Stokes equations with multiplicative noise, Stoch. Proc. Appl., 116 (2006), 1636-1659.

[44] R. Temam, Navier-Stokes Equations: Theory and Numerical Analysis, North Holland, 1979.

[45] J. B. Walsh, Finite element methods for parabolic stochastic PDE's, Potential Analysis, 23 (2005), 1-43. 
[46] X. Wan, X. Zhou and W. E, Study of the noise-induced transition and the exploration of the configuration space for the Kuramoto-Sivashinsky equation using the minimum action method, Nonlinearity, 23 (2010), 475-493.

[47] X. Wan, An adaptive high-order minimum action method, J. Compt. Phys., 230 (2011), 8669-8682.

[48] X. Wan and G. Lin, Hybrid parallel computing of minimum action method, Parallel Comput., 39 (2013), 638-651.

[49] X. Wan, A minimum action method for small random perturbations of twodimensional parallel shear flows, J. Compt. Phys., 235 (2013), 497-514.

[50] X. Wan, A minimum action method with optimal linear time scaling, Comm. Comput. Phys., 18(5) (2015), 1352-1379.

[51] X. Wan, B. Zheng and G. Lin, The hp-adaptivity of minimum action method, preprint.

[52] X. Wan, H. Yu and W. E, Model the nonlinear instability of wall-bounded shear flows as a rare event: A study on two-dimensional Poiseuille flow, Nonlinearity, 28 (2015), 1409-1440.

[53] X. Wan and X. Zhou, On small random perturbations of elliptic problems, preprint.

[54] Y. Yan, Galerkin finite element methods for stochastic parabolic partial differential equations, SIAM J. Numer. Anal., 43(4) (2005), 1363-1384.

[55] H. Yu, X. Wan and W. E, A stochastic study of nonlinear stability of $2 D$ Poiseuille flows, preprint.

[56] J. Zhang and Q. Du, Shrinking dimer dynamics and its applications to saddle point search, SIAM J. Numer. Anal., 50 (2012), 1899-1921.

[57] W. Zhang, T. Li and P. Zhang, Numerical study for the nucleation of onedimensional stochastic Cahn-Hilliard dynamics, Comm. Math. Sci., 10(4) (2012), 1105-1132.

[58] X. Zhou, W. Ren and W. E, Adaptive minimum action method for the study of rare events, J. Chem. Phys., 128 (2008), 104111.

[59] X. Zhou and W. E, Study of noise-induced transitions in the Lorenz system using the minimum action method, Comm. Math. Sci., 8(2) (2010), 341355 . 